\title{
Collapse of a liquid-saturated granular column on a horizontal plane
}

\author{
Alexis Bougouin $\odot,{ }^{*}$ Laurent Lacaze $\odot{ }^{\dagger}{ }^{\dagger}$ and Thomas Bonometti $\odot$ \\ Institut de Mécanique des Fluides de Toulouse (IMFT), Université de Toulouse, CNRS, Toulouse, France
}

(Received 23 December 2018; published 20 December 2019)

\begin{abstract}
Laboratory experiments on the collapse of a liquid-saturated granular column on a horizontal plane are reported. The trigger and the resulting dynamics of the collapse when occurring, as well as the shape of the final deposit, are characterized and analyzed in light of some dimensionless parameters, namely, the "column" Bond number Bo, the grain diameter to capillary length ratio $d / l_{c}$, the initial aspect ratio $a$, the Stokes number St, and the initial volume fraction $\phi$, by varying the properties of the interstitial fluid and of the grains, the geometry, and the compaction of the initial granular column. The main contribution of this study is to: (i) provide a diagram of the different regimes of collapse shown to be mostly controlled by capillary effect, (ii) develop simple criteria that capture the transitions between each regime in terms of critical values of the Bond number and the ratio of the grain diameter to the capillary length, (iii) extend a predictive model of the runout for dry collapses to the more general case of liquid-saturated granular collapses, and (iv) quantify the influence of $a, \mathrm{St}$, and $\phi$ on the collapse dynamics and the shape of the final deposit in the capillary-free regime. A perspective description of the role of the interstitial fluid on the spreading of the granular medium, and more particularly of the driving role of the fluid, is discussed and argued on the basis of the present set of experiments.
\end{abstract}

DOI: 10.1103/PhysRevFluids.4.124306

\section{INTRODUCTION}

Gravity-driven debris flows, composed of water-saturated granular materials, are encountered in many geophysical applications [1,2]. They are known to cause human and material damages due to their highly energetic dynamics. A particular feature of debris flows is that they can spread on longer distance than an equivalent dry situation [3]. The interstitial liquid then plays a major role on the dynamics of this grain-liquid mixture.

To understand the physical processes involved and controlling the dynamics of granular flows, laboratory experiments of canonical configurations turn out to be useful. For instance, the release of a dry granular column, initially at rest in a reservoir, on a horizontal or inclined bottom plane, referred to as the granular collapse, has shown to be a relevant configuration to mimic rockfalls, including the triggering of the avalanche, the flow dynamics, and the jamming process [4-9]. These dry granular collapses have been intensively studied through both axisymmetric [4,5] and quasi-2D $[6,7]$ geometries. In the latter configuration, the initial column is characterized by its length $L_{i}$ and its height $H_{i}$. When the granular column is suddenly released, the granular material spreads rapidly and then stops forming a final deposit characterized by its runout length $L_{f}$ and its final height $H_{f}$ (see Fig. 1). One of the remarkable feature of the granular collapse is that the length scales of the

\footnotetext{
*Present address: Université Clermont Auvergne, CNRS, IRD, OPGC, Laboratoire Magmas et Volcans, F-63000 Clermont-Ferrand, France; alexis.bougouin@uca.fr

†laurent.lacaze@imft.fr
} 

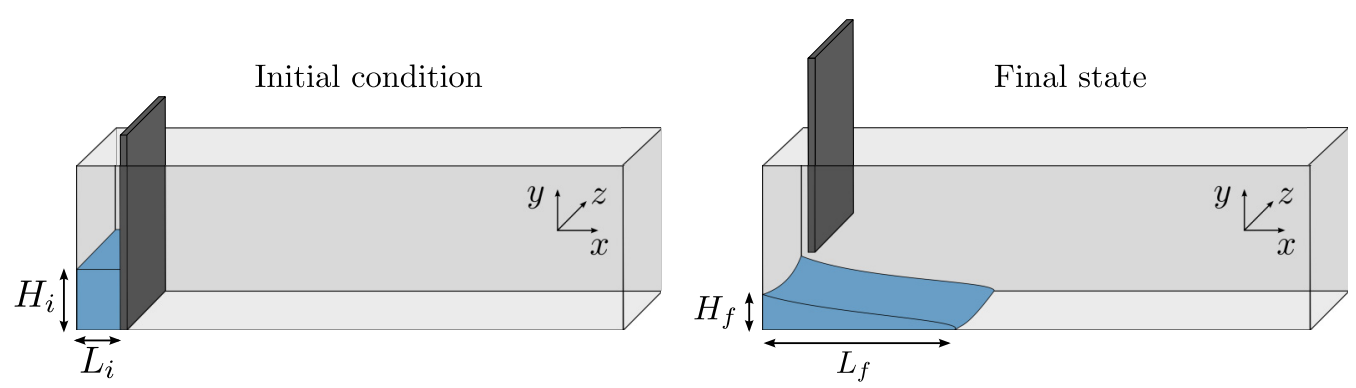

FIG. 1. Sketch of the experimental setup: A liquid-saturated granular column, defined by its initial length $L_{i}$ and its initial height $H_{i}$, initially on the left side of a sluice gate, is released into air and eventually forms a deposit characterized by the runout length $L_{f}$ and the final height $H_{f}$.

final deposit, when using an appropriate dimensionless form, mostly depend on the initial aspect ratio $a=H_{i} / L_{i}$ following simple power-laws.

In the present contribution, the configuration of the granular collapse in laboratory is extended to the case of a liquid-saturated granular material to mimic the situation of debris flows. To our knowledge, no study has considered this specific configuration contrary to the case of an unsaturated granular column, the so-called pendular state [10]. However, even in this case, studies mainly focused on the stability of the wet granular pile due to capillary effect as an analogy with the sand castle configuration [11-13], while the dynamics of the collapse beyond threshold has only been reported in a few studies [14-18]. In the latter case, these studies showed that the characteristics of the deposit, as the runout length, the final height, and the top and toe angles of the final deposit, are strongly dependent on the amount of liquid and the Bond number while time and velocity scales remain fairly similar to the dry configuration. Configurations where the amount of liquid becomes similar to the volume of grains, denoted capillary state and slurry state, still suffers from a lack of studies.

Other configurations share some similarities with the present liquid-saturated granular column, namely, the slumping of a neutrally buoyant suspension [19-23] and the fully immersed granular collapse [24-30]. In the first case, the density of the interstitial fluid and that of the grains is similar and hence the granular pressure is canceled. Contrary to a dry granular case, the collapse of a neutrally buoyant suspension column does not stop in most of the cases. However, the presence of the interface is shown to play a major role on the dynamics of the suspension, at least, at large initial volume fractions $\phi[22,23]$. In the case of a fully immersed granular collapse, the granular pressure exists as grains are denser than the surrounding fluid, and the jamming process is recovered. However, the interstitial liquid and the surrounding one are obviously the same and capillary effects are absent. In this configuration, three granular flow regimes are usually defined, namely, the freefall, inertial, and viscous regimes. These regimes are shown to be controlled by (i) the Stokes number St which prescribes the relative influence of the grain inertia and viscous dissipation of the fluid, and (ii) the grain-fluid density ratio $[28,31,32]$. Moreover, in the viscous regime, the initial volume fraction $\phi$ of the granular column has also been shown to play a significant role on the collapse as opposed to the dry case [24].

The liquid-saturated granular collapse at the laboratory scale then deserves a specific attention through an experimental study and some physical modeling. To highlight the similarities with the above-mentioned configurations as well as the key differences which could be significant for applications, the questions raised in the present contribution are the following. Is a liquid-saturated granular column affected by capillary effect as the unsaturated case? For instance, can the column support its own weight and remain static similar to the sand castle configuration? Can capillary effect influence the dynamics of the collapse beyond the aspect ratio reported in the dry configuration? Moreover, as capillary effect is expected to be strongly affected by the scale of the flow, can we 
then use the liquid-saturated granular configuration at the laboratory scale as a pertinent model for geophysical applications? Finally, what is the influence of the initial aspect ratio, the Stokes number and the volume fraction on the dynamics of a liquid-saturated granular collapse?

\section{EXPERIMENTAL DETAILS}

Laboratory experiments are performed in a horizontal transparent channel of rectangular crosssection. The dimensions are $2 \mathrm{~m}$ long, $0.35 \mathrm{~m}$ high, and $0.20 \mathrm{~m}$ wide along the streamwise $x$, vertical $y$, and spanwise $z$ coordinates, respectively (see Fig. 1). The origin $(x, y)=(0,0)$ is set at the bottom of a side wall of the channel. On this side, a finite volume reservoir is delimited by a sluice gate located at $x=L_{i}$. Four different reservoir widths are used, $L_{i}=3,6.5,10$, and $15 \mathrm{~cm}$. The reservoir is filled up to a height $H_{i}$ with a liquid-saturated granular material as explained in the following paragraphs. The initial height of the column $H_{i}$ is varied from 3 to $33 \mathrm{~cm}$, leading to an initial aspect ratio $a=H_{i} / L_{i}$ in the range $a=[0.2: 11]$.

The liquid-saturated granular column is composed of an interstitial fluid based on a mixture of water and Ucon oil 75H90000 (a polyalkylene glycol soluble in water) and spherical glass beads manufactured by Sigmund Lindner GmbH. The dynamic viscosity of the interstitial fluid depends on the relative concentration of Ucon oil and water which is characterized by the mass fraction $c_{m}=m_{o} /\left(m_{o}+m_{w}\right)$ with $m_{o}$ and $m_{w}$ the mass of oil and water, respectively. The mass fraction $c_{m}$ is varied from $0 \%$ (pure water) to $40 \%$ which corresponds to a variation of viscosity in the range $\eta_{f}=[1: 350] \mathrm{cP}$. The viscosity of the fluid mixture is obtained by rheometric measurements using a cone-plate geometry in a Haake Mars III rheometer with an accuracy of 5\%. The corresponding fluid density is in the range $\rho_{f}=[1000: 1064] \mathrm{kg} \mathrm{m}^{-3}$, measured by a DMA 35 Anton Paar electronic densimeter with an accuracy of $\pm 0.5 \mathrm{~kg} \mathrm{~m}^{-3}$. In some experiments, the influence of surface tension $\sigma$ has been investigated in the case of a pure water liquid, i.e., $c_{m}=0$, using $0.5 \mathrm{ml}$ of Triton X-100 per liter of water. This leads to a decrease of surface tension from $\sigma=$ $0.07 \mathrm{~N} \mathrm{~m}^{-1}$ to $\sigma=0.03 \mathrm{~N} \mathrm{~m}^{-1}$, whose values have been measured before the experiments using an electronic tensiometer DSA 100 with an accuracy of $5 \%$. Note that some dusty environment could slightly reduce the effective value of the surface tension during the experiments [33]. However, the quantitative bias would be similar for all experiments presented, which does not change the interpretation of the results. The grain density is $\rho_{p}=2500 \mathrm{~kg} \mathrm{~m}^{-3}$, the grain to interstitial liquid ratio being roughly constant and of order one. Its influence will thus be disregarded in the following. Different grain sizes are also investigated, the grain diameters ranging from $d=0.120 \mathrm{~mm}$ to $d=10 \mathrm{~mm}$. The corresponding angle of avalanche $\theta_{a}$ and angle of repose $\theta_{r}$ are given in Appendix A.

To prepare the initial liquid-saturated granular column, grains are poured in the reservoir previously filled with the liquid. The granular suspension is then mixed with a propeller driven by a drill motor. Then, the reservoir is gently kicked with a rubber mallet to compact the granular material [24,34]. The maximum volume fraction of the initial column obtained here is $\phi=0.64 \pm 0.02$, i.e., a dense packing configuration. Even if most of the results presented in the following correspond to this dense packing configuration, some results will be presented for a range of volume fraction $\phi=[0.616: 0.643]$. Finally, the excess of fluid standing above the granular column is removed by a valve located under the reservoir. The valve is closed when the height of the liquid is equal to that of the top of the granular column. Note that the volume of interstitial liquid varies with $\phi$ as $(1-\phi) H_{i} L_{i} W$ with $W=0.2 \mathrm{~m}$ the width of the channel. This procedure suggests that the liquid-saturated granular column is at the frontier between the slurry state and capillary state as top surfaces of the grains are just in contact with the liquid interface. The range of dimensionless parameters, associated with the properties of the liquid-saturated granular mixture together with the ambient air and covered in the present study, are given in Table I.

At time $t=0$, the sluice gate is removed manually at a roughly constant speed of $2 \mathrm{~m} \mathrm{~s}^{-1}$ and the initial liquid-saturated granular column is released on a smooth bottom plane. In all of the experiments, the corresponding opening time of the sluice gate was less or equal to the 
TABLE I. Dimensionless parameters associated with the properties of the liquid-saturated granular column, used in the present work. Note that the indices $p, f$, and $a$ denote the grain, the interstitial liquid, and the ambient air, respectively. $V_{\text {grains }}$ and $V_{\text {column }}$ denote the volume of grains and the total volume of the column, respectively.

\begin{tabular}{lcc}
\hline \hline Dimensionless parameter & Definition & Covered range \\
\hline Grain-liquid density ratio & $\rho_{p} / \rho_{f}$ & $\approx 2.5$ \\
Liquid-air density ratio & $\rho_{f} / \rho_{a}$ & $10^{3}$ \\
Liquid-air viscosity ratio & $\eta_{f} / \eta_{a}$ & {$\left[55: 2 \times 10^{4}\right]$} \\
Initial aspect ratio & $a=H_{i} / L_{i}$ & {$[0.2: 11]$} \\
Initial volume fraction & $\phi=V_{\text {grains }} / V_{\text {column }}$ & {$[0.616: 0.643]$} \\
\hline \hline
\end{tabular}

column free-fall timescale $T_{\mathrm{FF}}=\left(2 H_{i} / g\right)^{1 / 2}$ which ensures that the initial condition does not affect significantly the collapse, as shown by Ref. [35] (see their Sec. 4.1). Once released, the material deforms then, it slumps in the majority of cases. Its shape is characterized by the height profile $h(x, t)$, extracted from image analysis using a classical shadowgraphy method. For this purpose, the 2D-flow in the $(x, y)$ plane is recorded using a Basler $2048 \times 2048$ pixels camera or a sCMOS Lavision $2560 \times 2160$ pixels camera and a backlight source on the opposite side of the channel. The obtained resolution is about $400 \mu \mathrm{m} /$ pixel and the acquisition rate is between 10 and $200 \mathrm{~Hz}$, depending on the timescale of the flow.

\section{PRELIMINARY OBSERVATIONS: FLOW REGIMES}

\section{A. The different flow regimes in a water-saturated case}

The typical evolution of a water-saturated granular collapse for various grain diameters, namely, $d=120 \mu \mathrm{m}, d=500 \mu \mathrm{m}$, and $d=5 \mathrm{~mm}$, is presented in Fig. 2. Here, the initial aspect ratio and the initial volume fraction are identical for all cases, with $a \approx 1.3\left(H_{i}=20 \mathrm{~cm}, L_{i}=15 \mathrm{~cm}\right)$ and $\phi \approx 0.64$, respectively. Movies of these experiments are also available in the Supplemental Material [36]. One can clearly observe that the collapse dynamics and the morphology of the final deposit are strongly dependent on the size of the grains.

At small $d=120 \mu \mathrm{m}$ [Fig. 2(a)], the liquid-grain column remains static after the sluice gate is opened (even after several hours). This observation has already been reported in the case of a neutrally buoyant suspension, with $a=1, \phi>0.61$, and $d<230 \mu \mathrm{m}$ [23]. This configuration will

(a)

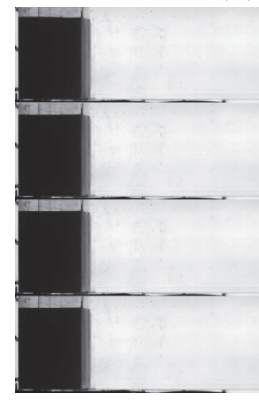

(b)

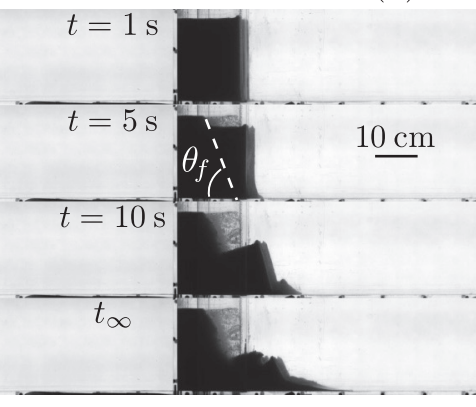

(c)

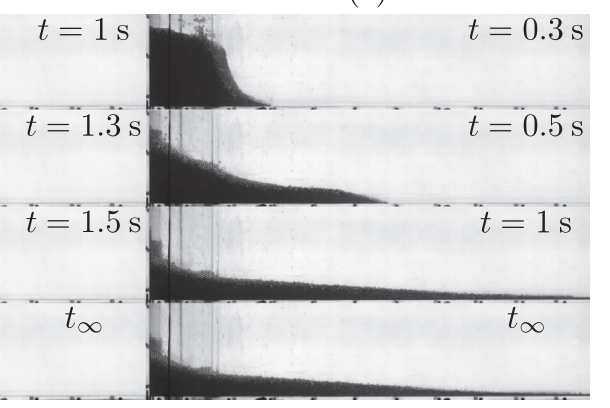

FIG. 2. Snapshots of some water-saturated granular collapses for various grain diameters: (a) $d=120 \mu \mathrm{m}$, (b) $d=500 \mu \mathrm{m}$, and (c) $d=5 \mathrm{~mm}$. In Fig. 2(b), the failure angle $\theta_{f}$ with respect to the horizontal plane is indicated. In all cases, the initial aspect ratio is $a \approx 1.3$ and the initial volume fraction is $\phi \approx 0.64$ (i.e., initially densely packed). 


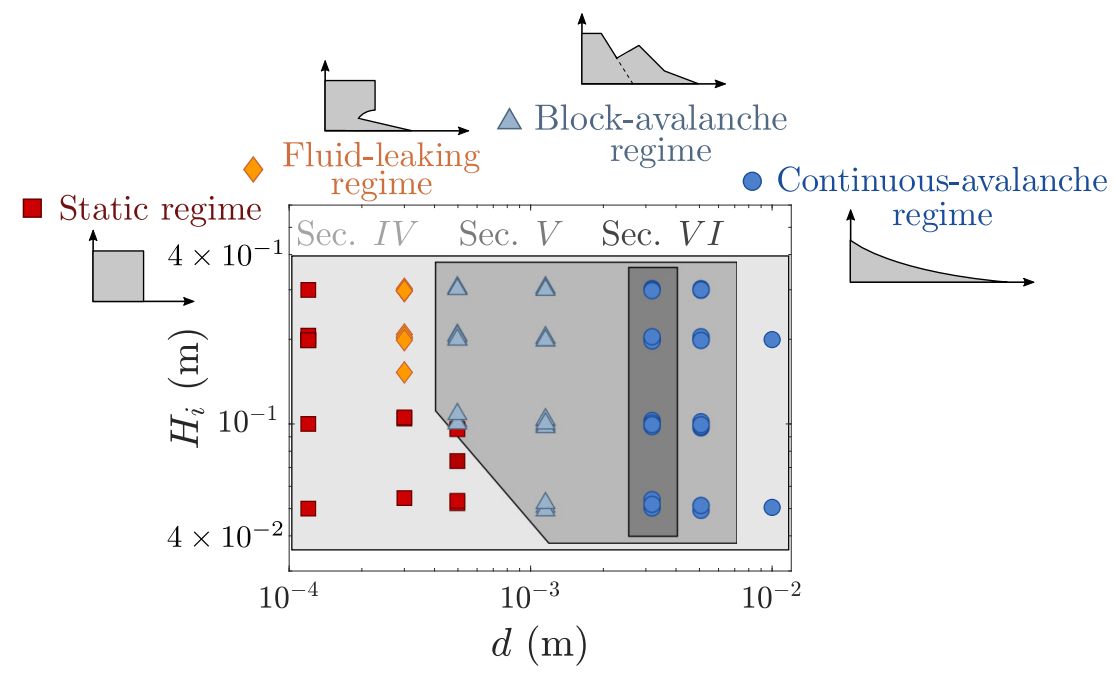

FIG. 3. Diagram of the different regimes of collapse of an initially densely packed water-saturated granular column in the parameter space $\left(d, H_{i}\right)$ with $L_{i}=[3: 15] \mathrm{cm}$.

be referred to as the static regime. At larger $d=300 \mu \mathrm{m}$ (not shown here), the granular column remains roughly static while the interstitial liquid flows out of the porous granular medium. This induces a slight erosion at the bottom of the granular column, while the upper part maintains its initial shape. This configuration will be referred to as the fluid-leaking regime. A movie of this regime is available in the Supplemental Material with $a \approx 2$ [36]. At even larger $d=500 \mu \mathrm{m}$ [Fig. 2(b)], the granular material cannot be maintained in its initial shape and the upper part of the column collapses as a block. This leads to a non-monotonous height profile of the final deposit. This configuration will be referred to as the block-avalanche regime. Note that, in this case, a failure angle (w.r.t. the horizontal plane) can be defined [see Fig. 2(b)]. It is observed to be roughly constant, namely, $\theta_{f}=56 \pm 3^{\circ}$, independently of the grain diameter $d$ and the initial height $H_{i}$ in the range of parameters considered here. At the largest $d=5 \mathrm{~mm}$ presented here [Fig. 2(c)], the water-saturated granular column collapses, the motion being initiated at the bottom of the column as usually observed in the dry configuration. The granular column then rapidly spreads in the horizontal direction and then stops forming a final deposit. This configuration will be referred to as the continuous-avalanche regime. Even if the two latter regimes highlight significant differences, they can be both considered as a collapse regime as, in both cases, part of the material slumps and spreads over the horizontal bottom. Note that the block-avalanche regime and the continuous-avalanche regime have already been observed for cohesive granular collapses [16].

These different regimes are summarized in the $\left(d, H_{i}\right)$ plane in Fig. 3. In particular, the static regime, fluid-leaking regime, block-avalanche regime, and continuous-avalanche regime are represented by red squares $(\square)$, orange diamonds $(\diamond)$, gray triangles $(\triangle)$, and blue circles $(\bullet)$, respectively.

\section{B. Dimensional analysis}

Results obtained in the literature with an equivalent dry configuration did not report the different regimes observed here, suggesting that the initial aspect ratio $a$ and the initial volume fraction $\phi$ are not the pertinent dimensionless parameters to explain these observations. Then, the interstitial fluid has to be accounted for to explain these regimes. Three dimensionless parameters, involving the properties of the interstitial fluid and of the interface between the interstitial liquid and the ambient 
air, can be defined for this configuration, namely, the Stokes number St, the length ratio $d / l_{c}$ (with $l_{c}$ the capillary length, defined later in this section), and the Bond number Bo.

The Stokes number is defined as $\mathrm{St}=(1 / 18 \sqrt{2})\left[\rho_{p}\left(\rho_{p}-\rho_{f}\right) g d^{3}\right]^{1 / 2} / \eta_{f}$, and it prescribes the relative influence of the grain inertia and fluid dissipation. Here, this number is in the range $\mathrm{St}=$ $[0.2: 200]$. Note that with the present values of $\left(\mathrm{St}, \rho_{p} / \rho_{f}\right)$ the dynamics of the collapse may be in the transition region between the viscous regime and the inertial regime, characterized in fully immersed granular flows [28,31].

The length ratio $d / l_{c}$ and the Bond number Bo account for possible capillary effects at the interface between the interstitial liquid and the ambient air. In the present case, these contributions can only take place close to the free-surface of the liquid-saturated granular medium, contrary to the pendular state configuration for which capillary bridges can also induce cohesion in the bulk of the granular medium. To activate a capillary pressure on the granular material in the present configuration, the liquid-air interface has to be deformed at a length scale which is similar to the capillary length $l_{c}$. This might occur on a relatively short timescale, as such deformation is not present in the initial configuration. This suggests an initial decompaction of the granular column just after removing the sluice gate, leading to a so-called capillary state for which deformation of the liquid-air interface occurs on a scale imposed by the grain diameter. Note that this decompaction only occurs for initially dense configuration, typically $\phi \gtrsim 0.6$, for which the granular media need to expand before flowing [24]. Due to this initial deformation of the system, a capillary pressure induced by the curvature of the liquid-air interface between two consecutive grains at the surface of the granular medium can prevent extra grain motion. A first dimensionless parameter that can be built accounting for this capillary effect is based on the ratio between the grain diameter $d$ and the capillary length $l_{c}=\sqrt{\sigma / \rho_{f} g}$, i.e., $d / l_{c}=d \sqrt{\rho_{f} g / \sigma}$. Here, it is found in the range $d / l_{c}=[0.04: 4]$, which suggests that capillary effects could affect the grain motion at the interface. To compare the macroscopic scale $H_{i}$ and the microscopic scale $d$ highlighted in Fig. 3, another dimensionless number can be built upon the pressure due to the weight of the initial column and the capillary pressure. The capillary pressure induced by the interface deformation and driven at the grain scale can be estimated as $P_{c} \approx \varepsilon \sigma / d$, where $\varepsilon$ quantifies the size of the meniscus compared to the diameter $d$. Additionally, the driving mechanism for the collapse of the column is the static pressure of the grain-fluid mixture. Considering the effective medium, this pressure can be estimated as $P_{h} \approx \rho g H_{i}$, where $\rho=\phi \rho_{p}+(1-\phi) \rho_{f}$ is the effective density of the grain-fluid column. The ratio of these two pressures gives $P_{h} / P_{c}=\rho g H_{i} d / \varepsilon \sigma$. This ratio is analogous to a Bond number, which includes both the "small" and the "large" scales of the problem, namely, the grain diameter $d$ and the initial height of the column $H_{i}$. In the following and for the sake of simplicity, we withdraw $\varepsilon$ from the previous expression and here define the so-called "column" Bond number as $\mathrm{Bo}=\rho g H_{i} d / \sigma$. In this work, the Bond number is in the range $\mathrm{Bo}=[1: 600]$ which suggests that capillary effects could affect the collapse.

The definitions of $\mathrm{St}, d / l_{c}$, and Bo all involve $d$, suggesting that they could be associated with the existence of the different regimes as highlighted by the dimensional transition map in Fig. 3. However, previous results on fully immersed granular collapses did not report these regimes. Yet, fully immersed collapses also involve St even when no liquid-air interface is present. Actually, viscous dissipation can affect the dynamics of the flow but cannot explain plastic transition as fluid viscosity does not involved an extra pressure contribution. This suggests that $\mathrm{St}$ is probably not the pertinent parameter to provide a physical interpretation to the regime transitions, even if it can play a significant role on the dynamics of the system within a specific regime, as discussed later. Moreover, note that these regimes remind of the sand castle configuration often reported for unsaturated granular column [11-13]. These observations suggest that capillarity, i.e., $d / l_{c}$ and Bo, controls the different regimes and more particularly their transition.

To conclude, the transition between the different regimes can only be due to capillary effect and thus involves $d / l_{c}$ and Bo. The role of $d / l_{c}$ and the "column" Bond number Bo regarding these transitions of regimes is thus discussed through simplified models, in Sec. IV. Then, the dynamics of the collapse regimes can be analyzed with respect to the above-mentioned dimensionless 


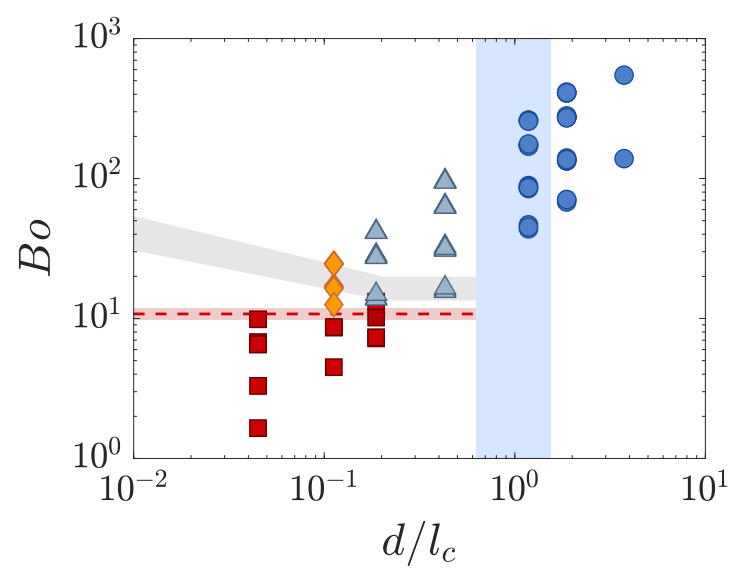

FIG. 4. Diagram of the different regimes of collapse of an initially densely packed water-saturated granular column in the parameter space $\left(d / l_{c}\right.$, Bo $)$ : $\square$ static; fluid-leaking; $\Delta$ block-avalanche; continuousavalanche. Red dashed line: Eq. (1) with $\varepsilon=5.5 \pm 0.5$; gray band: Eq. (2) with $\varepsilon=5.5, \phi=0.64$ and $22^{\circ} \leqslant \delta \leqslant 28^{\circ}$; blue area: region where $d_{c} / l_{c} \leqslant d / l_{c} \leqslant d_{c}^{\prime} / l_{c}$ with $d_{c} / l_{c}$ and $d_{c}^{\prime} / l_{c}$ given by Eqs. (3) and (4), respectively.

parameters, namely, Bo, $a$, St, $\phi$. For the sake of clarity, the description of the dynamics of the collapse regimes, i.e., block-avalanche regime and continuous-avalanche regime, in which capillary action can remains significant at least close to the transition between these two regimes, will first be discussed with respect to the Bond number Bo and the initial aspect ratio $a$ in the case of a water-saturated configuration (Sec. V). Then, a deeper investigation of the continuous-avalanche regime, when capillary effect becomes negligible, is presented in Sec. VI, with a specific attention on the influence of the aspect ratio $a$, the Stokes number St and the initial volume fraction $\phi$. For this purpose, the water-saturated configuration is extended to the liquid-saturated granular collapse for which the viscosity of the interstitial liquid is varied from the water one. In Fig. 3, shaded areas summarize the regimes associated to the following sections.

\section{TRANSITIONS OF REGIMES: ROLE OF THE BOND NUMBER AND OF THE GRAIN DIAMETER TO CAPILLARY LENGTH RATIO}

Capillary effect is assumed to be at the origin of the different regimes observed in Fig. 3 as explained in the previous section. We therefore report the experimental data of Fig. 3 in the parameter space $\left(d / l_{c}\right.$, Bo) in Fig. 4. These two dimensionless numbers have been discussed in the previous section, and basically characterize the capillary effect at the grain and column scales, respectively. In Fig. 4, one observes that $d / l_{c}$ and Bo are indeed the relevant dimensionless parameters controlling the transitions. More particularly, Bo shows to be the control parameter which prescribes the transition between the static regime and others regimes. This transition is therefore based on an equilibrium state at the column scale. In contrast, $d / l_{c}$ delineates the two collapsing regimes, which transition is thus controlled by the grain length scale. To provide simple scalings highlighting the influence of capillary effect on the transitions between the different regimes, we first assert that the static regime can exist as long as capillary effect at the interface between the interstitial liquid and the surrounding air prevents from the initial decompaction of the granular medium that would be necessary for motion. In return, the static granular medium holds the interstitial fluid back due to the same capillary effect. Therefore, motion can be initiated either by the fluid (fluid-leaking regime) or both the grains and the fluid (avalanche regimes). It is found here that both situations are obtained even if collapsing regimes cover a wider zone in the parameter space $\left(d / l_{c}\right.$, Bo), while the fluid-leaking regime is only observed in a delimited area (see 
Fig. 4). In the present work, the above-mentioned motions can only be initiated by a driving pressure associated with gravity.

\section{A. Static regime versus fluid-leaking regime}

Let us consider the only liquid phase inside the column to address the transition from the static regime to the fluid-leaking regime. Here, the granular phase can be regarded as a static porous medium filled with water as the granular materials remain at rest, unlike collapsing regimes. Its presence is thus mostly reduced to the existence of a capillary pressure on the interstitial fluid phase at its interface with the ambient air, i.e., top and right of the column in Fig. 1. The hydrostatic pressure of the liquid $P_{h}^{f}$ is maximum at the bottom wall and one can write $P_{h}^{f}=\rho_{f} g H_{i}$. In the static regime, this pressure is not large enough to overcome the capillary pressure $P_{c}$, which prevents the liquid from flowing out of the granular column, or the equivalent static porous media in the present model. As mentioned in the previous section, this pressure is estimated as $P_{c}=\varepsilon \sigma / d$ where $\varepsilon=\mathcal{O}(10)[12,37]$. Now, we assume that a rough criterion for the transition between the static regime and the fluid-leaking regime is when $P_{h}^{f}=P_{c}$. Using this equality together with the definition of the Bond number Bo $=\rho g H_{i} d / \sigma$, one obtains the following criterion for the transition between the static regime and the fluid-leaking regime as

$$
\mathrm{Bo}=\varepsilon \frac{\rho}{\rho_{f}} \equiv \mathrm{Bo}^{F} .
$$

We find that a value of $\varepsilon=5.5 \pm 0.5$ allows to delimit the transition obtained experimentally. With the present set of liquid and grains, it corresponds to $\mathrm{Bo}^{F}=11$. In Fig. 4, the dashed red line corresponds to $\varepsilon=5.5$, while the light red area around this value indicates the sensitivity of the transition with the parameter $\varepsilon$ in the range \pm 0.5 . From now on and unless stated otherwise, we therefore set $\varepsilon=5.5$.

\section{B. Static regime versus block-avalanche regime}

Let us now consider the only granular medium. The fluid phase is reduced to the only existence of a capillary pressure at the interface of the granular medium with the ambient air, as well as a buoyancy contribution to the weight of the granular material. We propose a simple model based on a failure criterion determined by the Mohr-Coulomb theory. For this purpose, one assumes that the granular column is subjected to a vertical stress $\sigma_{y}$ and a horizontal stress $\sigma_{x}$ which obey the following failure relation $\sigma_{y}^{c}=[(1+\sin \delta) /(1-\sin \delta)] \sigma_{x}$, where the exponent $c$ stands for "critical" and $\delta$ is the friction angle of the granular material. In the case of a liquid-saturated granular column, both the granulostatic pressure $P_{g}=\phi\left(\rho_{p}-\rho_{f}\right) g H_{i}$ and the capillary pressure $P_{c}=\varepsilon \sigma / d$ located at the interface of the column, induce a stress at the base of the granular column. In the horizontal direction, this reads $\sigma_{x}=\varepsilon \sigma / d$, while in the vertical direction, one can write $\sigma_{y}=\phi\left(\rho_{p}-\rho_{f}\right) g H_{i}+\varepsilon \sigma / d$, at the bottom wall. Note that we have assumed here that the capillary stress is fully transmitted to the granular media. It then leads to the following criterion for the transition between the static regime and the block-avalanche regime,

$$
\mathrm{Bo}=\frac{\varepsilon \rho}{\phi\left(\rho_{p}-\rho_{f}\right)}\left(\frac{2 \sin \delta}{1-\sin \delta}\right) \equiv \mathrm{Bo}^{G} .
$$

In Fig. 4, the gray band indicates the location of the critical Bond number $\mathrm{Bo}^{G}$ given by Eq. (2). The thickness of the line represents the range of $\mathrm{Bo}^{G}$ considering a friction angle included in the range $\theta_{r} \leqslant \delta \leqslant \theta_{a}$, with $\theta_{r}$ and $\theta_{a}$ the repose and avalanche angles of the granular material, respectively. Note that these angles are $d$-dependent as accounted for by Eq. (A1) (see Appendix A), leading to a variation of the transition with $d / l_{c}$ in the parameter space $\left(d / L_{c}\right.$, Bo) (Fig. 4). Equation (2) allows us to delineate the block-avalanche regime fairly well. It is worth mentioning that for small $d / l_{c}$, this transition occurs at larger Bo. It therefore allows the emergence of a fluid-leaking regime zone 
in the parameter space $\left(d / l_{c}\right.$, Bo), as observed in Fig. 4 and described previously. To finish with, note that the Mohr-Coulomb theory gives an estimate of the failure angle $\theta_{f}$ of the granular column (w.r.t. the horizontal direction), which reads $\theta_{f}=\pi / 4+\delta / 2$. Assuming that the friction angle $\delta$ is between $\theta_{r}=22^{\circ}$ and $\theta_{a}=28^{\circ}$, for $500 \mu \mathrm{m} \leqslant d \leqslant 1.15 \mathrm{~mm}$, we obtain $56^{\circ} \lesssim \theta_{f} \lesssim 59^{\circ}$, which is in agreement with experimental observations, i.e., $\theta_{f}=56 \pm 3^{\circ}$ [see Fig. 2(b), for instance].

\section{Block-avalanche regime versus continuous-avalanche regime}

The above-mentioned criteria are based on the scale of the entire granular column. The key idea in the following is to suppose that a continuous-avalanche regime, in contrast to the block-avalanche regime, can only be obtained when capillary forces cannot influence any longer the grain motion. Thus, considering a simple balance at the grain scale between gravitational forces, of the order of $\rho_{p} g d^{3}$, and capillary forces, of the order of $\sigma d$, leads to a criterion based on a critical diameter to capillary length ratio $d_{c} / l_{c}$ as

$$
\frac{d_{c}}{l_{c}}=\left(\frac{\rho_{f}}{\rho_{p}}\right)^{1 / 2},
$$

which here gives $d_{c} / l_{c} \approx 0.6$. A more "refined" analysis consists in considering the academic problem of a local capillary bridge between two grains. In the specific case of two identical spheres in contact, a reasonable estimate of the capillary force is $F_{c} \approx \pi \sigma d$ [38]. Equating $F_{c}$ with the weight of one sphere $F_{g}=\pi \rho_{p} g d^{3} / 6$ gives a second criterion for existence of a continuous-avalanche regime in terms of a slightly different critical length ratio $d_{c}^{\prime} / l_{c}$ as

$$
\frac{d_{c}^{\prime}}{l_{c}}=\left(\frac{6 \rho_{f}}{\rho_{p}}\right)^{1 / 2},
$$

which here gives $d_{c}^{\prime} / l_{c} \approx 1.6$. In Fig. 4, the blue area represents the range $d_{c} / l_{c} \leqslant d / l_{c} \leqslant d_{c}^{\prime} / l_{c}$ and shows a fair prediction of the transition between the block-avalanche regime and the continuousavalanche regime. Note that the critical diameter has been shown to be dependent on other parameters, such as the grain roughness, the liquid content or the meniscus shape, for instance, which are not consider here $[39,40]$.

To conclude, the simple scaling analyses proposed here and based on a significant contribution of capillary effects at the column [Eqs. (1) and (2)] and grain [Eqs. (3) and (4)] scales allowed to explain the transition between the different observed regimes, from static to avalanche dynamics.

\section{AVALANCHE REGIMES: BEYOND THE INITIAL ASPECT RATIO}

In the block-avalanche and the continuous-avalanche regimes, i.e., in any collapse regime, one may attempt to characterize the deposit in terms of final runout length $L_{f} / L_{i}$ and height $H_{f} / H_{i}$, as done in the case of dry granular collapses.

Figure 5 presents the corresponding $L_{f} / L_{i}$ [Fig. 5(a)] and $H_{f} / H_{i}$ [Fig. 5(b)] as a function of the initial aspect ratio $a=H_{i} / L_{i}$. Note that, in the present work, $L_{f}$ is defined as the mean value of four estimates obtained with various criteria presented in Appendix B. The observed general trend here is an increase of $L_{f} / L_{i}$ and a decrease of $H_{f} / H_{i}$ with $a$. However, one can observe a significant dispersion of results, unlike the equivalent dry case. In the latter case, the data fall reasonably well on some master curves which indicates that the final runout length and height follow simple power-laws over $a$ (see cross symbols in Fig. 5, corresponding to the dry results). This dispersion is, among others, related to capillary effect via the specific value of the Bond number being varied here either through $H_{i}$ or $d$. This statement is confirmed by the coherent evolution of $L_{f} / L_{i}$ and $H_{f} / H_{i}$ with respect to Bo, as highlighted by the gray levels attributed to symbols. In particular, $L_{f} / L_{i}$ and $H_{f} / H_{i}$ are shown here to increase and decrease with Bo, respectively. Note that $L_{f} / L_{i}$ roughly increases by a factor of 3 when $a$ is increased over a decade and Bo is increased by about three decades. Similar conclusions can also be drawn with the final height $H_{f} / H_{i}$. 

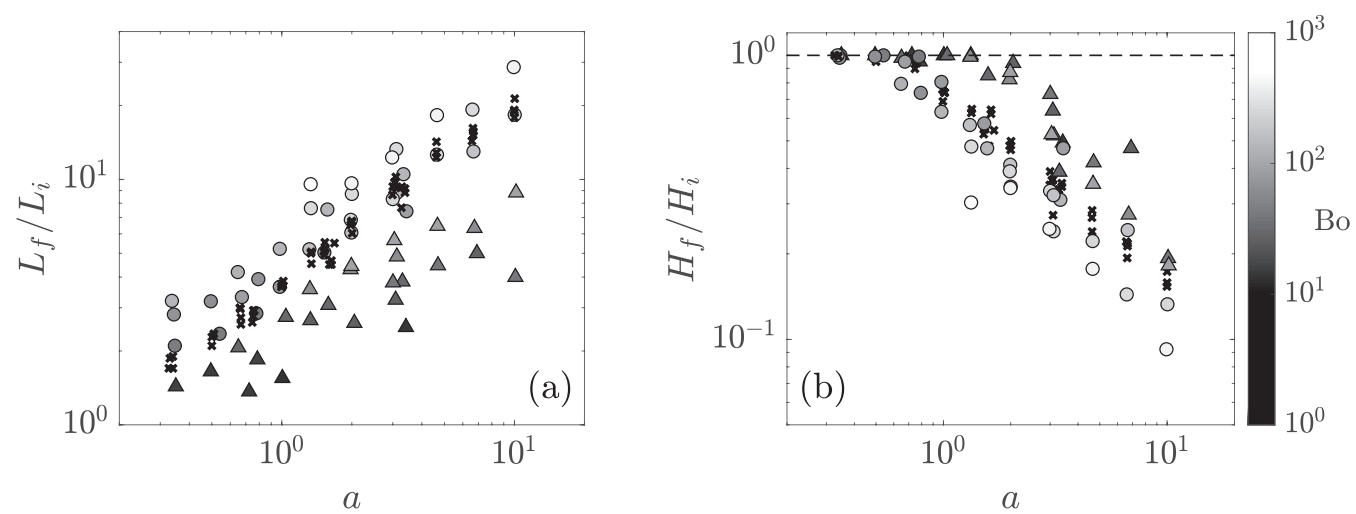

FIG. 5. (a) Runout length $L_{f} / L_{i}$ and (b) final height $H_{f} / H_{i}$ as a function of the initial aspect ratio $a=$ $H_{i} / L_{i}$. Symbols correspond to experiments performed in the block-avalanche regime $(\triangle)$ and in the continuousavalanche regime (O), while colors indicate the value of the Bond number Bo $=\rho g H_{i} d / \sigma$ (see color bar). Cross symbols correspond to the dry case with the same set of grains.

Using an approach similar to Lajeunesse et al. [7] who predicted the power-law dependence between $L_{f}$ and $a$ for a dry granular collapse, we develop a model accounting for Bo-effects in the case of a liquid-saturated granular configuration. For conciseness, the detailed derivation of the model is given in Appendix C.

Briefly, we first perform a force balance on the liquid-saturated granular column, including (i) the friction force at the bed level, (ii) a force related to the granulostatic pressure, and (iii) the capillary force at the grain scale. Note that such force balance model provides a description of the fluid-particle mixture but it neglects the relative motion of the two phases and the viscous dissipation induced by the liquid. We further assume the shape of the final deposit to be triangular, which can be anticipated to be a crude assumption, in particular, to described the block-avalanche regime. Using mass conservation, we finally obtain the following equation:

$$
\frac{L_{f}-L_{i}}{L_{i}}=4 \lambda^{2} a^{2}\left(\frac{L_{f}}{L_{i}}\right)^{-2}-2 \lambda^{2} \mu \phi \frac{\rho_{p}-\rho_{f}}{\rho} a-2 \varepsilon \lambda^{2} \mu \frac{a}{\mathrm{Bo}}\left(\frac{L_{f}}{L_{i}}\right),
$$

which predicts the final runout length $L_{f}$ as a function of the density ratio $\left(\rho_{p}-\rho_{f}\right) / \rho$, the initial aspect ratio $a$, the initial volume fraction $\phi$, the "column" Bond number Bo and two extra parameters $\mu$ and $\lambda$. The parameter $\mu$ is the friction coefficient between the granular material and the bottom plane and $\lambda$ is an empirical parameter defined as the ratio of the characteristic time of the collapse to the free-fall time. In particular, we found $\lambda \approx 4.8$ (for more details, see Appendix $C$ ). Note that, at Bo $\gg 1$ and $a \ll 1$, Eq. (5) gives $\left(L_{f}-L_{i}\right) / L_{i} \propto a$, while at Bo $\gg 1$ and $a \gg 1$, Eq. (5) gives $\left(L_{f}-L_{i}\right) / L_{i} \approx L_{f} / L_{i} \propto a^{2 / 3}$. We thus recover Lajeunesse et al. [7]'s scaling laws obtained for a dry granular collapse.

The experimental results, already given in Fig. 5(a), are compared to Eq. (5) in Fig. 6(a). In particular, the final runout $L_{f} / L_{i}$ is shown in the parameter space $(a$, Bo). Symbols and full lines correspond to experimental data and solutions of Eq. (5), respectively, and colors indicate ranges of $L_{f} / L_{i}$. In Fig. 6(b), $L_{f} / L_{i}$ is also plotted as a function of Bo, with $a=2$, to provide a more quantitative comparison. To highlight the influence of the different terms in Eq. (5) and to adjust fitting parameters of the model $(\lambda, \mu$, and $\varepsilon$ ) when needed, partial solutions corresponding to different balance of the terms in Eq. (5) are presented in Fig. 6 (dash-dotted and dashed lines). First, dash-dotted lines correspond to a balance of the driving pressure term [first term on the right-hand side of Eq. (5)] and the friction term induced by the capillary pressure [third term on the right-hand side of Eq. (5)]. Here, $\varepsilon \mu=2.2$, or equivalently $\varepsilon=5.5$ and $\mu=0.4$, has been chosen according to the results obtained in the previous section. Dashed lines correspond to the balance of 


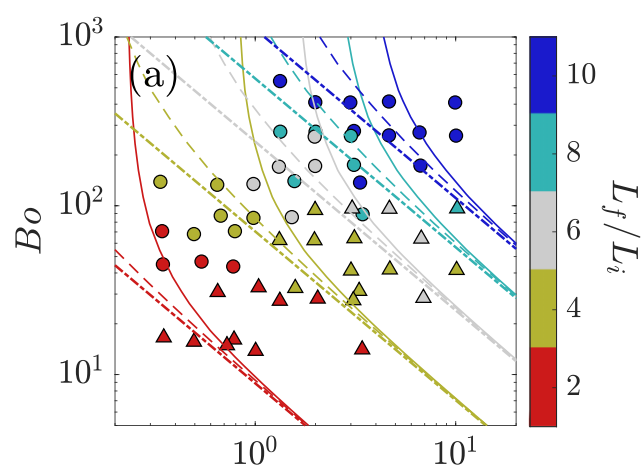

$a$

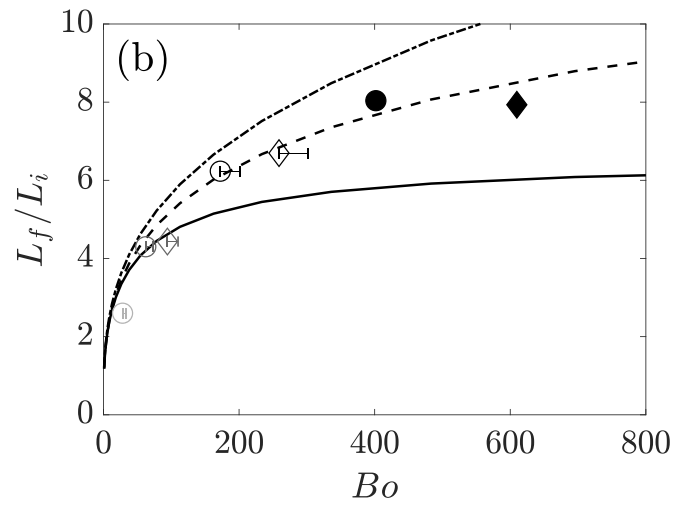

FIG. 6. (a) Runout length $L_{f} / L_{i}$ in the parameter space ( $a$, Bo). Experiments: $(\triangle)$ block-avalanche regime, (O) continuous-avalanche regime. (b) Runout length $L_{f} / L_{i}$ as a function of Bo, with $a=2$. The Bond number is varied through the grain size $d$ ( $d$ increasing from light to dark gray), the initial height of the granular column $H_{i}=[20,30] \mathrm{cm}$ (circles and diamonds, respectively) and the surface tension $\sigma \sim[0.03,0.07] \mathrm{N} \mathrm{m}^{-1}$ (closed and opened symbols, respectively). Predictions of Eq. (5): (- . . -) balance of the driving pressure term and the friction term induced by capillary pressure [first and third terms on the right-hand side of Eq. (5)] with $\varepsilon \mu=2.2$, i.e., $\varepsilon=5.5$ and $\mu=0.4\left(\approx \tan \theta_{r}\right) ;(--)$ inertialess solution with $\lambda \rightarrow \infty, \varepsilon \mu=2.2$ and $\mu=0.04$; ( - ) full solution with $\lambda=4.8, \varepsilon \mu=2.2$, and $\mu=0.04$. The initial volume fraction is set to $\phi=0.64$.

the right-hand side of Eq. (5), corresponding to $\lambda \rightarrow \infty$, i.e., an inertialess solution. Here, $\mu$ has to be set to $\mu=0.04$, while keeping $\varepsilon \mu=2.2$ as previously, to adjust the solution with experimental data [see the dashed lines in Fig. 5(b), in particular]. To finish with, solid lines correspond to the full solution of Eq. (5) keeping parameters of the right-hand side terms the same as previously and setting $\lambda=4.8$.

The present model is in qualitative agreement with experiments. In particular, the increase of $L_{f} / L_{i}$ with $a$ and Bo is captured by the present model. However, the parametric study (dashed, dash-dotted and solid lines, in Fig. 6) shows that the values of the parameters have to be adjusted to reasonably fit the experimental solutions. Here, we chose to modify only the friction term associated with the weight of the column $[\mu=0.04$ in the second term on the right-hand side of Eq. (5)] and to keep $\varepsilon \mu=2.2$ and $\lambda=4.8$ as found in the previous section and from the estimation of the collapse timescale (Appendix C), respectively. Choosing this set of parameters allows to capture most of the dynamics of the collapse, as observed in Fig. 6. However, adding the inertial term does not provide better results than a simple balance of the right-hand side terms in Eq. (5). Better adjustment of the fitting parameters $\lambda, \mu$, and $\varepsilon$ could give better agreement between the model and experimental data. Regarding the simplicity of the model, we preferred to keep most of the parameters as the one obtained through other methods for the sake of clarity. In any case, the value of $\lambda=4.8$, as obtained in Appendix C, does not provide satisfactory solution whatever $\mu$ and $\varepsilon$. This can be attributed to the simplicity of the model and, in particular, the relatively gross modeling of the inertial term. Moreover, other physical ingredients included in St and $\phi$ have been disregarded so far. These parameters probably influence the dynamics of the collapse, and could also explained some of the discrepancies between the model and the experimental data. These parameters will be discussed in detail in the next section for which capillary effects can be disregarded, allowing to distinguish their specific influence from Bo.

Overall, the present results show that the simple model Eq. (5) is able to predict the general evolution of the final deposit shape of a liquid-saturated granular collapse as a function of $a$ and Bo, meaning that most of the physical mechanisms is included in the model. However, caution must be exercised when using Eq. (5) for a quantitative prediction, since it is sensitive to the specific value 


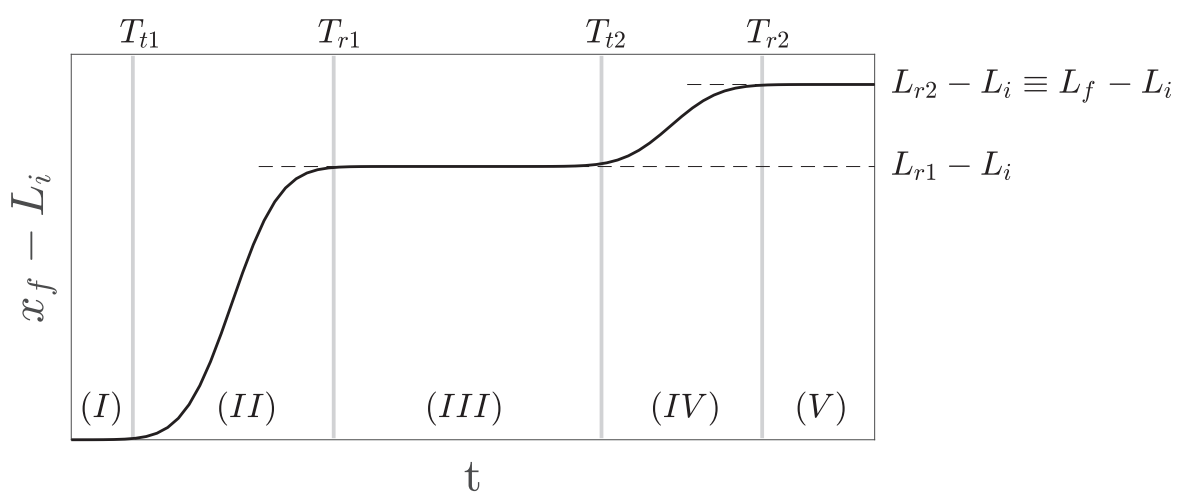

FIG. 7. Sketch of the temporal evolution of the granular front position $x_{f}-L_{i}$ for a liquid-saturated granular collapse in the continuous-avalanche regime. The dynamics is decomposed into five stages: three stages where the front of the column is at rest $(I, I I I$, and $V)$ and two stages where the front is spreading (II and $I V)$. Indices $t$ and $r$ used in the definition of the transition times and distances denote "trigger" and "rest", respectively.

of the parameters $\lambda, \mu$ and $\varepsilon$. Improvement of the model would deserve a specific attention which is beyond the scope of the present paper.

\section{CONTINUOUS-AVALANCHE REGIME: THE CAPILLARY-FREE REGIME}

In previous sections, the influence of capillary effects has been shown to play a major role on the dynamics and the characteristics of the final deposit of a water-saturated granular collapse. These observations and descriptions are of interest for many applications as well as for improving our knowledge on the physical processes involved in liquid-grain mixture flows. However, in many geophysical applications, the flow is generally not affected by the capillary effects highlighted here, which are likely to be observed due to a scale effect. In this section, we thus focus on the continuousavalanche regime in which capillary effects are negligible (Bo $\gg 1$ and $d>d_{c}$ ). In this case, beyond the initial aspect ratio $a$, the Stokes number St and the initial volume fraction $\phi$ could also influence the flow dynamics as observed and reported in different studies on immersed granular collapses $[24,28]$. In particular, in the case of debris flows, Rickenmann [3] reported that the presence of liquid could enhance mobility compared with an equivalent dry situation. This is probably one of the key issue to be addressed for prediction purpose. The spreading length will thus be compared with the dry configuration and the influence of the different dimensionless parameters will discussed in this section. As often reported in the literature, its corresponding dry runout length, denoted here $L_{f}^{d}$, will be assumed to be only dependent on $a$ and not affected by St or $\phi$. This latter statement will nevertheless be discussed in the following.

Recall that the liquid-saturated granular collapse belongs to the continuous-avalanche regime at the laboratory scale if the grain diameter is larger than a critical value (see Sec. IV). To observe and quantify the influence of the Stokes number, different liquid viscosities have thus to be explored to reach small values of St for large enough $d$ to remain in the continuous-avalanche regime. A first observation when St is varied in the continuous-avalanche regime is a modification of the front evolution on a relatively large timescale compared to the case of a water-saturated granular column presented so far. A sketch of the temporal evolution of the front position $x_{f}-L_{i}$ is shown in Fig. 7. First, the front remains nearly frozen from $t=0$ to $t=T_{t 1}$ referred to as the trigger time (phase $I$ ). At $t=T_{t 1}$, the granular column collapses until reaching a first runout length $L_{r 1}$, at $t=T_{r 1}$ (phase $I I$ ). For $T_{r 1} \leqslant t \leqslant T_{t 2}$ (phase $I I I$ ), the granular material remains frozen, with a front position equal to the location of the first stop $x_{f}=L_{r 1}$. More surprisingly, and contrary to other collapse configurations as the dry or the fully immersed one, at $t=T_{t 2}$, the granular front 

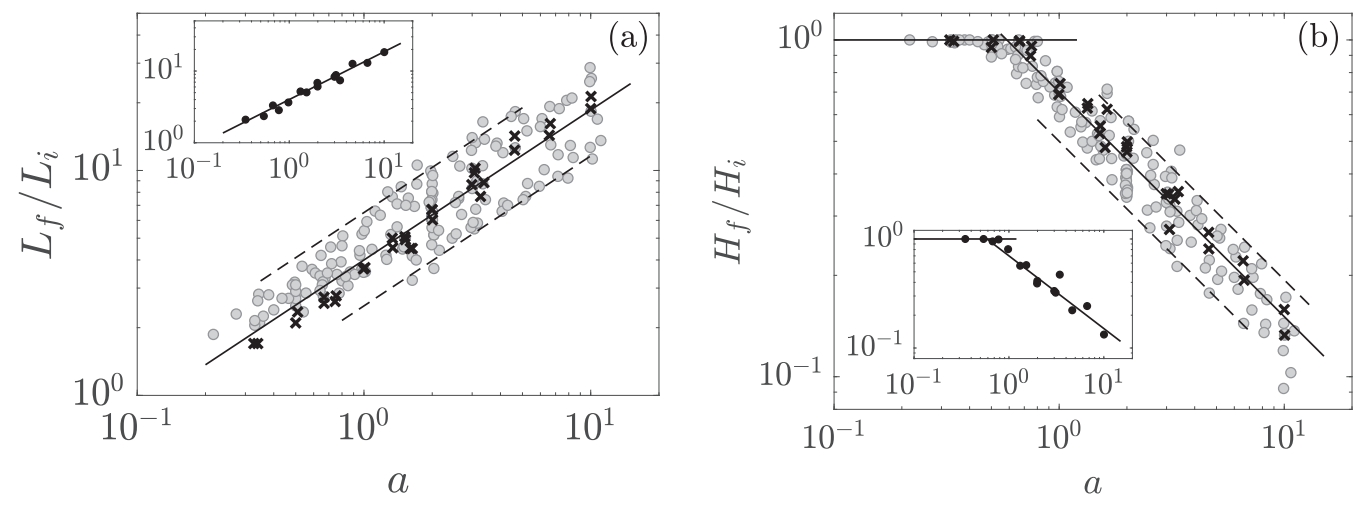

FIG. 8. (a) Runout length $L_{f} / L_{i}$ and (b) final height $H_{f} / H_{i}$ as a function of the initial aspect ratio $a=H_{i} / L_{i}$ for liquid-saturated granular collapses in the continuous-avalanche regime $(0)$. Note that here, the Stokes number St and the initial volume fraction $\phi$ were varied. Inset: selected experiments where $\mathrm{St}=42$ and $\phi=0.63 \pm 0.01$. For comparison, we also plot the results of dry collapses $(\mathbf{x})$ performed with the same experimental setup and the same grains $(d=3.15$ and $5.0 \mathrm{~mm})$. (一) $L_{f} / L_{i}=\lambda_{1} a^{2 / 3}$, with $\lambda_{1}=4$ and $H_{f} / H_{i}=\lambda_{2} a^{-2 / 3}$ with $\lambda_{2}=0.7 ;(--) \lambda_{1}=[2.5: 6.5]$ and $\lambda_{2}=[0.5: 0.9]$.

spreads again due to liquid flowing out of the porous granular medium (phase $I V$ ). This liquid leaking dynamics leads to a modification of the shape of the deposit characterized by a second runout length $x_{f}=L_{r 2}$, at $t=T_{r 2}$ (phase $V$ ). Note that the runout length considered in previous sections corresponds to $L_{f}=L_{r 2}$. In the following, the different times $T_{t, r}$ are defined as the time for which we have $d x_{f}\left(T_{t, r}-\Delta t\right) / d t<\zeta$ and $d x_{f}\left(T_{t, r}\right) / d t \geqslant \zeta$ where $\Delta t$ is the time step between two consecutive images and $\zeta$ is a small threshold parameter of about $10 \%$ of $\sqrt{g H_{i}}$.

\section{A. Role of the initial aspect ratio}

Figure 8 presents the runout length $L_{f} / L_{i}$ [Fig. 8(a)] and the final height $H_{f} / H_{i}$ [Fig. 8(b)] as a function of the initial aspect ratio $a=H_{i} / L_{i}$ for liquid-saturated granular collapses in the continuous-avalanche regime (closed circles). For comparison, we also plot experimental results of dry collapses performed with the same experimental setup and the same grains (crosses). One first observes that $L_{f} / L_{i}$ and $H_{f} / H_{i}$ follow the same global trend over $a$ compared to the dry case, even if a significant dispersion in the results is observed. To highlight the origin of this dispersion, which cannot be longer attributed to capillarity as in previous sections, the same characteristic lengths are shown in the insets of Fig. 8 for a limited set of experiments where $\mathrm{St}=42$ and $\phi=0.63 \pm 0.01$. In this case, the dispersion is significantly reduced. This supports the fact that $\mathrm{St}$ and $\phi$ play a role on the characteristics of the final deposit, and thus explain the dispersion observed in the obtained measures of $L_{f} / L_{i}$ and $H_{f} / H_{i}$ shown in Fig. 8.

Moreover, the solid lines correspond to the scaling laws $L_{f} / L_{i}=4 a^{2 / 3}$ [Fig. 8(a)] and $H_{f} / H_{i}=$ $0.7 a^{-2 / 3}$ [Fig. 8(b)] in line with the usual scalings found for the dry situation [7]. Note that a best fit of the dry case gives $L_{f} / L_{i} \propto a^{0.73}$ and $H_{f} / H_{i} \propto a^{-0.70}$. Results obtained for the characteristic lengths of the final deposit of liquid-saturated granular collapses, when St and $\phi$ are varied, spread in an interval which remains bounded in the range of parameters considered here. In particular, this interval can be delimited by two scaling laws which share common features with the scaling laws obtained for the dry case, only the prefactor being modified (see the dashed lines in Fig. 8). More surprisingly, $L_{f} / L_{i}$ and $H_{f} / H_{i}$ can be either larger or smaller than in the dry case. This indicates the ambivalent role of the interstitial fluid on the granular material dynamics.

We can conclude that, beyond the initial aspect ratio $a$, for which the trend obtained in the dry case can be extrapolated to the present configuration, St and $\phi$ also play a role on the dynamics, 

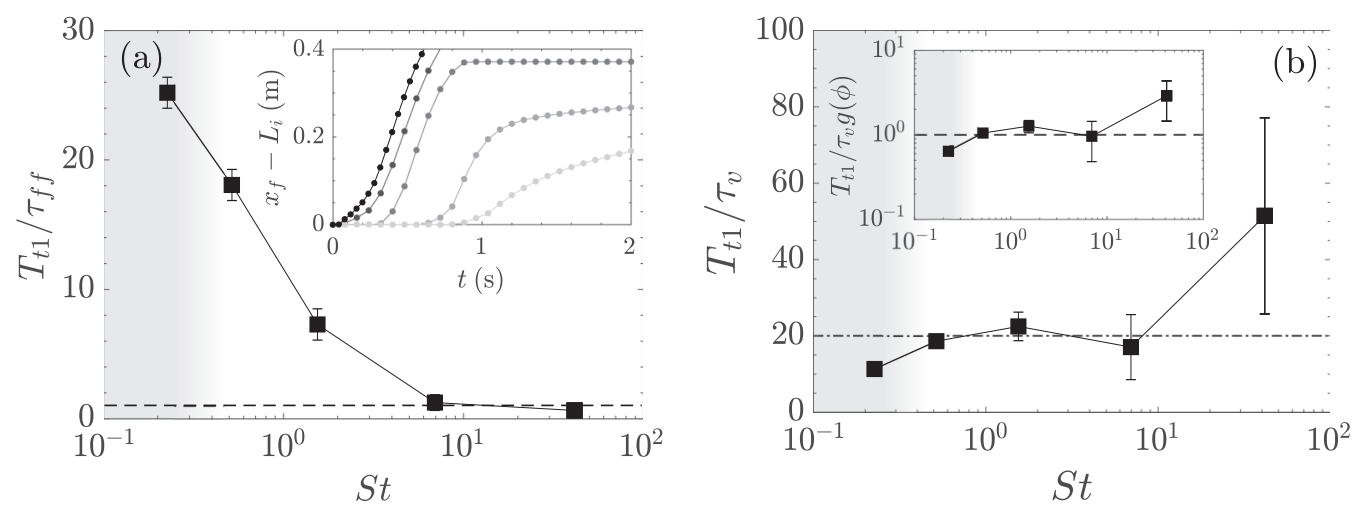

FIG. 9. Trigger time $T_{t 1}$ normalized by (a) the free-fall time $\tau_{\mathrm{ff}}=\left[2 \rho_{p} d /\left(\rho_{p}-\rho_{f}\right) g\right]^{1 / 2}$ and (b) the viscous time $\tau_{v}=18 \eta_{f} /\left(\rho_{p}-\rho_{f}\right) g d$ as a function of the Stokes number St $(a=2$ and $\phi=0.64)$. Insets: (a) Time evolution of the front position $x_{f}-L_{i}$ of the corresponding experiments. Colors indicate the range $\mathrm{St}=[0.2$ : 42] from light to dark gray. (b) Dimensionless trigger time $T_{t 1} / \tau_{v} g(\phi)$, with $g(\phi)=(1-\phi)^{-2.8}$ and $\phi=0.64$, as a function of St. (- - ) $T_{t 1} / \tau_{\mathrm{ff}}=T_{t 1} / \tau_{v} g(\phi)=1 ;(-\cdots) T_{t 1} / \tau_{v}=20$. The gray area represents the range of St for which no second spreading phase was observed.

thus modifying quantitatively the characteristic lengths of the final deposit. Their influences are characterized independently in the following sections.

\section{B. Role of the Stokes number}

To assess the influence of St, the other dimensionless parameters, $a, \phi$, and Bo are kept constant here. The variation of St is here obtained by varying the viscosity of the interstitial fluid $\eta_{f}$ while the other physical parameters are kept constant, namely, $d=3.15 \mathrm{~mm}, \rho_{p}=2500 \mathrm{~kg} \mathrm{~m}^{-3}$ and $\rho_{f} \approx$ $1000 \mathrm{~kg} \mathrm{~m}^{-3}$. We then set $a=2, \phi=0.64$ and $\mathrm{Bo}=160$. The influence of St is discussed in the following in terms of the timescales of the collapse (Figs. 9 and 10) and the shape of the final deposit (Figs. 11 and 12).

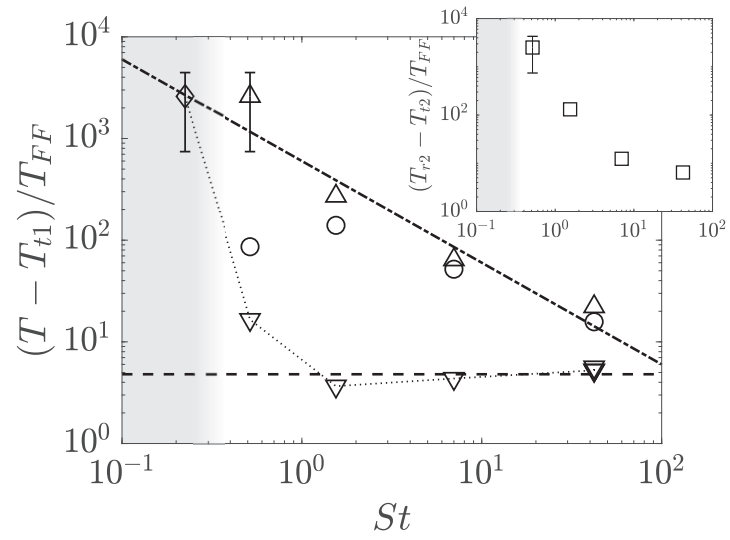

FIG. 10. Characteristic times of the front dynamics as a function of St $(a=2$ and $\phi=0.64):(\nabla) T_{r 1}-$ first stop, (O) $T_{t 2}$-second start, $(\triangle) T_{r 2}$-second stop. We substracted from these times the first trigger time $T_{t 1}$ and scaled them by the column free-fall timescale $T_{\mathrm{FF}}=\left(2 H_{i} / g\right)^{1 / 2} .(--)\left(T-T_{t 1}\right) / T_{\mathrm{FF}}=\lambda=4.8,\left(-\cdots-{ }_{-}\right)$ $\left(T-T_{t 1}\right) / T_{\mathrm{FF}} \propto \mathrm{St}^{-1}$. The gray area represents the range of $\mathrm{St}$ for which no second spreading phase was observed. The inset shows $\left(T_{r 2}-T_{t 2}\right) / T_{\mathrm{FF}}$ as a function of St. 


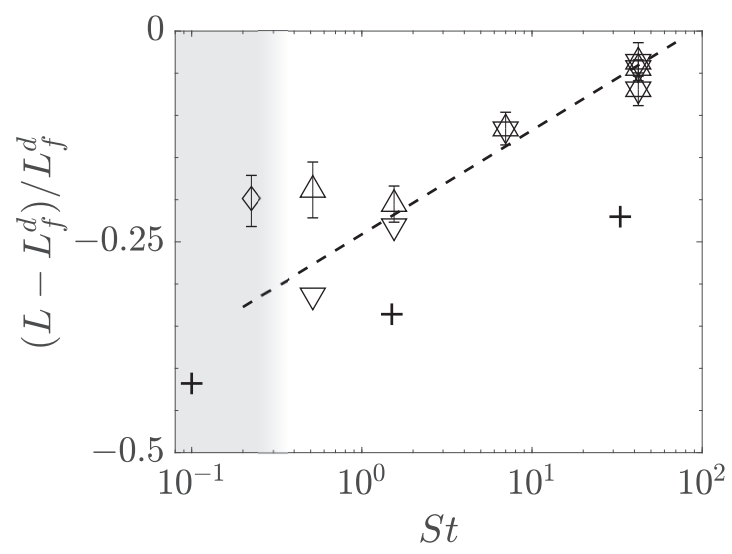

FIG. 11. Location of the first arrest $L_{r 1}(\nabla)$ and the second arrest $L_{r 2} \equiv L_{f}(\triangle)$ relative to that of the corresponding dry collapse $L_{f}^{d}$ as a function of St $(a=2$ and $\phi=0.64)$. (+) Results of the runout length $L_{f}^{i}$ for fully immersed dense granular collapses, extracted from Bougouin and Lacaze [28]. The gray area represents the range of St for which no second spreading phase was observed.

We first observe that a trigger lag time $T_{t 1}$ has to be reached prior collapse [see inset of Fig. 9(a)]. $T_{t 1}$ may correspond here to the time for the granular medium to expand prior to collapse, since the granular column is initially densely packed (recall that $\phi=0.64$ ). Such an observation has already been reported in the case of a fully immersed granular collapse [24,28,41]. The trigger time $T_{t 1}$ is plotted as a function of the Stokes number St in Fig. 9. Here, $T_{t 1}$ is normalized by the free-fall timescale at the grain scale, namely, $\tau_{\mathrm{ff}}=\left[2 \rho_{p} d /\left(\rho_{p}-\rho_{f}\right) g\right]^{1 / 2}$, which corresponds to the time that a grain needs to travel over a diameter $d$, starting from rest and submitted to gravity [Fig. 9(a)]. $T_{t 1} / \tau_{\mathrm{ff}}$ decreases for St increasing until reaching a limit value $T_{t 1} / \tau_{\mathrm{ff}} \approx 1(---)$. This observation is consistent with the idea that there is some viscous dissipation during the expansion of the granular media. This effect becomes negligible, at large St, where the trigger time $T_{t 1}$ is similar to the free-fall timescale $\tau_{\mathrm{ff}}$. Here, one can reasonably say that, for $\mathrm{St} \lesssim 1.5$, the expansion of the granular material is controlled by viscous dissipation while, otherwise, viscous dissipation can be neglected on the initial dynamics of the collapse.

Using a similar approach, a viscous timescale can be defined as $\tau_{v}=18 \eta_{f} /\left(\rho_{p}-\rho_{f}\right) g d$, which corresponds to the time that a grain needs to travel over a diameter $d$ under the action of gravity and
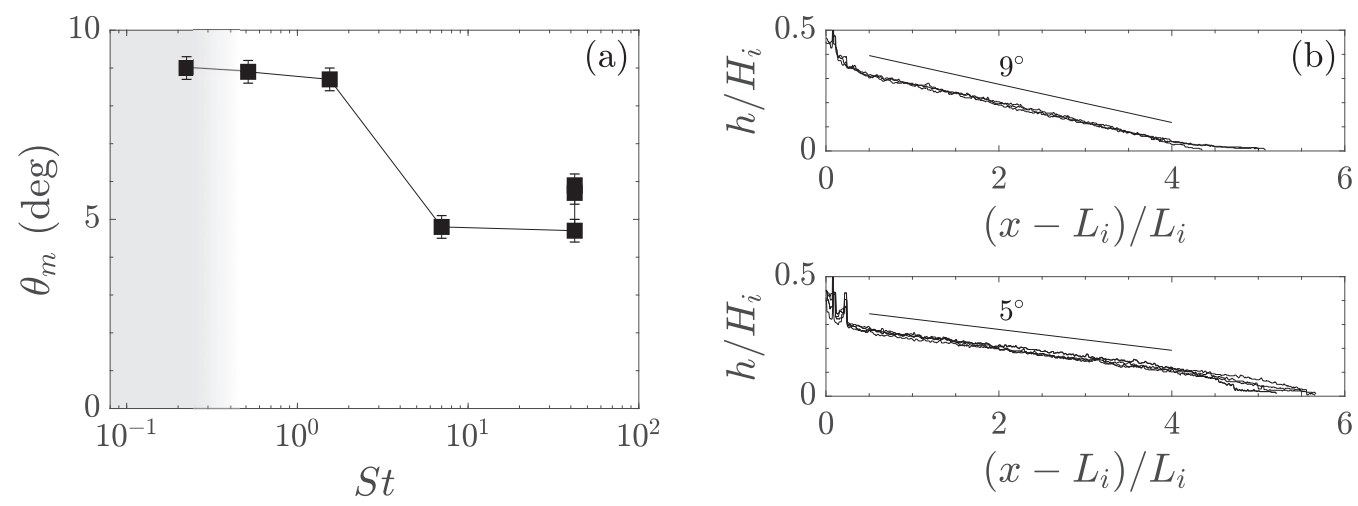

FIG. 12. (a) Mean angle $\theta_{m}$ as a function of the Stokes number St ( $a=2$ and $\left.\phi=0.64\right)$. The gray area represents the range of St for which no second spreading phase was observed. (b) Height profile $h / H_{i}$ as a function of $\left(x-L_{i}\right) / L_{i}$ for St $\lesssim 1.5$ (top) and $\mathrm{St} \gtrsim 7$ (bottom). 
submitted to a viscous drag force. Figure 9 (b) shows $T_{t 1} / \tau_{v}$ as a function of St. At low St, $T_{t 1} / \tau_{v}$ is actually constant and equal to $T_{t 1} / \tau_{v} \approx 20\left(-\cdots-\right.$-). It supports that the trigger time $T_{t 1}$ scales with a viscous time $\tau_{v}$ at the grain scale. Note that a better scaling is obtained considering an effective viscosity accounting for the local volume fraction. In particular, an effective viscosity of the form $\eta_{\text {eff }}=\eta_{f} g(\phi)$ with $g(\phi)=(1-\phi)^{-2.8}$ leads to $T_{t 1} / \tau_{v} g(\phi) \sim 1$, at sufficiently low St [dashed line in the inset of Fig. 9(b)]. The function $g(\phi)$ used here was obtained by Gibilaro et al. [42] in the case of a fluidized bed configuration. The initiation of the collapse is therefore controlled by the local interaction of the grain with the surrounding fluid, in particular, through an effective viscosity including both the viscosity of the surrounding fluid $\eta_{f}$ and the volume fraction $\phi$. From now on, $T_{t 1}$ is considered as the initial time of the collapse dynamics and timescales will thus be discussed accordingly.

We now focus on the time for the granular medium to reach its final rest state. As mentioned previously, a simple characterization of this timescale is more complicated than in the dry case as the final state is reached on different stages characterized by three different distinct times $T_{r 1}, T_{t 2}$, and $T_{r 2}$ (see Fig. 7). Figure 10 shows these different timescales as a function of St. Here, the quantities are made dimensionless using the free-fall timescale defined at the column scale $T_{\mathrm{FF}}=\left(2 \mathrm{H}_{i} / g\right)^{1 / 2}$. The dimensionless total time prior rest state $\left(T_{r 2}-T_{t 1}\right) / T_{\mathrm{FF}}$, i.e., the total time of the dynamics inducing grain motion, scales as $\mathrm{St}^{-1}$ (upward triangles and diamond). This indicates that the total time of motion scales with a viscous timescale since $T_{\mathrm{FF}} \mathrm{St}^{-1} \equiv 2\left(T_{\mathrm{FF}} / \tau_{\mathrm{ff}}\right) \tau_{v}$. Moreover, for $\mathrm{St} \approx 0.2$ (diamond), no second spreading phase (phase $I V$ in Fig. 7) was observed, i.e., $T_{r 1}$ and $T_{r 2}$ cannot be distinguished, and the collapse dynamics is mostly controlled by viscous dissipation (gray area). Otherwise, at larger St, the dynamics splits into two distinguished front motions, phase $I I$ and phase $I V$ as labeled in Fig. 7, that will be referred to as "first collapse" and "second collapse", respectively. The dimensionless time of the "first collapse" presents a sharp transition around St $\approx$ 1.5 (downward triangles). More specifically, for $\mathrm{St} \lesssim 1.5,\left(T_{r 1}-T_{t 1}\right) / T_{\mathrm{FF}}$ rapidly decreases with increasing St while, for $\mathrm{St} \gtrsim 1.5$, the time of the phase $I I$ is roughly constant and scales as the free-fall timescale $T_{\mathrm{FF}}$, as usually obtained for dry granular collapses and dam-break flows with a single fluid phase [7,28,43]. In particular, we find $\left(T_{r 1}-T_{t 1}\right) / T_{\mathrm{FF}} \approx \lambda=4.8(---)$. Interestingly, for $\mathrm{St} \geqslant 1.5$, the duration of the "first collapse" slightly increases for increasing St which suggests a possible influence of the interstitial fluid yet (we elaborate on this in the following). In the inset of Fig. 10, the "second collapse" timescale $\left(T_{r 2}-T_{t 2}\right) / T_{\mathrm{FF}}$ decreases with St on the whole range considered here, i.e., for St $\lesssim 10^{2}$. This second dynamics is therefore always controlled by viscous dissipation supporting that the final time of the collapse scales with a viscous time. Finally, it is likely that, for $\mathrm{St} \gtrsim 10^{2}, T_{r 1}$ and $T_{r 2}$ cannot be distinguished and the collapse dynamics is only controlled by inertia. No "second collapse" will be observed in agreement with the fact that viscous dissipations are neglected.

The different spreading lengths corresponding to these different timescales are now considered. For this purpose, one defines the spreading lengths of the "first collapse" and "second collapse" relative to that of the corresponding dry collapse in their dimensionless form, i.e., $\left(L-L_{f}^{d}\right) / L_{f}^{d}$ with $L \equiv L_{r 1}$ and $L \equiv L_{r 2}$ for the "first collapse" and the "second collapse", respectively. Figure 11 presents $\left(L-L_{f}^{d}\right) / L_{f}^{d}$ as a function of St. For comparison, the case of the fully immersed granular collapse, $L \equiv L_{f}^{i}$, for initially densely packed column (i.e., $\phi=0.64$ ), extracted from Bougouin and Lacaze [28], is also shown (crosses). A first observation is that $L_{f}^{i} \leqslant\left(L_{r 1}, L_{r 2}\right) \leqslant L_{f}^{d}$ for the range of St considered here. Also, both spreading lengths basically increase with increasing St, which is consistent with the decrease of viscous dissipation due to the interstitial fluid. Additionally, at $\mathrm{St} \approx 1.5$, a sharp transition of the evolution of $L_{r 2}$ with $\mathrm{St}$ is obtained, which corresponds to the range for which the "first collapse" timescale switches from a viscous regime to an inertial regime (see Fig. 10). More specifically, for St $\gtrsim 1.5, L_{r 1} \approx L_{r 2}$, indicating that the fluid flow draining out of the granular medium during the "second collapse", does not significantly modify the runout length of the granular material. Most of the granular medium thus spreads during the "first collapse". In contrast, for $\mathrm{St} \lesssim 1.5$ (diamond in Fig. 11), $L_{r 1}$ decreases with decreasing St down to $\mathrm{St} \approx 0.2$, 
under which no second spreading phase was observed, i.e., $L_{r 1}=L_{r 2}$, while $L_{r 2}$ remains constant, such as $\left(L_{r 2}-L_{f}^{d}\right) / L_{f}^{d} \approx-0.2$. A significant difference between the "first collapse" and the "second collapse", i.e., between the "first collapse" and the final rest state, is therefore obtained in the range $0.5 \lesssim \mathrm{St} \lesssim 1.5$. To finish with, it is worth noticing that even if $\left(L_{r 1}, L_{r 2}\right) \leqslant L_{f}^{d}$ for the range of St considered here, i.e., $0.2<\mathrm{St}<42$, the observed trend seems to indicate that $L_{r 1}=L_{r 2}$ reaches $L_{f}^{d}$ at around $\mathrm{St} \approx 10^{2}$.

The transition from a viscous regime to an inertial regime with increasing St can also be observed in the morphology of the deposit which may be characterized by the angle of the deposit. The mean angle $\theta_{m}$ of the height profile is observed to get two distinct values depending on St, with in particular, $\theta_{m} \approx 5^{\circ}$, at large St, and $\theta_{m} \approx 9^{\circ}$, at low St [see Fig. 12(a)]. These values are smaller than the angle of repose and they may depend on the initial aspect ratio $a$, as already reported in dry and fully immersed granular collapses [5,24,28]. Moreover, at low St, the liquid-saturated case leads to a smaller value of the mean angle, i.e., $\theta_{m} \approx 9^{\circ}$, compared to the corresponding fully immersed case for which $\theta_{m} \approx 17^{\circ}$ (from [28]), supporting, once again, the very different influence of the liquid phase between these two configurations. While the viscous-inertial transition is clearly obtained around $\mathrm{St} \approx 1.5$ when considering the St-evolution of $\theta_{m}$, the St-effects reported previously in each regime is hardly observed. To highlight the different shapes of the deposit in these two regimes, Fig. 12(b) shows the height profile $h / H_{i}$ as a function of $\left(x-L_{i}\right) / L_{i}$, for $\mathrm{St} \lesssim 1.5$ (top) and for $\mathrm{St} \gtrsim 7$ (bottom). The deposit is thinner and longer at large St in line with the results of Fig. 11.

We can thus summarize the above-mentioned results as follows. For $\mathrm{St}<\mathrm{St}_{c 1}$ with $\mathrm{St}_{c 1} \approx 0.2$, a single viscous-dominated collapse is obtained. Otherwise, for $\mathrm{St}>\mathrm{St}_{c 1}$, the dynamics splits into two "collapses", leaving a "transitory rest state" between them. For $\mathrm{St}<\mathrm{St}_{c 2}$ with $\mathrm{St}_{c 2} \approx 1.5$, both the "first collapse" and the "second collapse" are mostly controlled by viscous dissipation. As a consequence, the timescales and the first spreading length are strongly dependent on St, while the final deposit is roughly unchanged. This suggests a complex influence of the interstitial fluid which can act both as a dissipative process on the granular flow, as in the case of the fully immersed situation, and as a driving force on the granular material when the latter stops in a state of non-equilibrium for a liquid phase (i.e., with an inclined height profile). $\mathrm{St}_{c 2}$ has been highlighted to be a dominant critical value of the transition from a viscous-dominated regime towards an inertial-dominated regime, with in particular, a sudden change of the mean angle of the height profile observed on the final deposit. For St $>\mathrm{St}_{c 2}$, the "first collapse" is mostly inertial, while the "second collapse" remains in a viscous regime. However, the "second collapse" does not affect significantly the granular deposit and the granular material is therefore spread during the "first collapse". In contrast to the dry case, the timescale of the "first collapse" and the final spreading length slowly evolve with St. The latter observation is a signature of an inertial-type (by opposition to viscous) contribution of the interstitial fluid phase. Finally, for larger St, say $\mathrm{St}>10^{2}$, it is expected to recover a single inertia-dominated collapse for which the timescale and the final deposit are similar to the dry case, i.e., for which the dynamics is only controlled by the granular phase.

\section{Role of the volume fraction}

As observed in Fig. 8(a), the spreading length of a liquid-saturated collapse can overcome that of the dry collapse. However, the range of parameters used in the previous section (by only varying St for an initially dense packing, i.e., $\phi=0.64$ ) did not allow to observe such a situation, even if the trend at large St suggested that such situation could be reached. As the influence of $\phi$ can be significant for the immersed configuration, we now focus on the influence of the initial volume fraction $\phi$ of the granular column, at a relatively large $S t \approx 42$, i.e., close to a possible change of sign of $L_{f}-L_{f}^{d}$, with $L_{f}$ being the final spreading length. In particular, we investigate the dynamics and the final deposit of the granular material. Here, the properties of the liquid and the grains are fixed and only the initial volume fraction $\phi$ is varied. More particularly, the interstitial fluid is water and the size of grains is $d=3.15 \mathrm{~mm}$. 

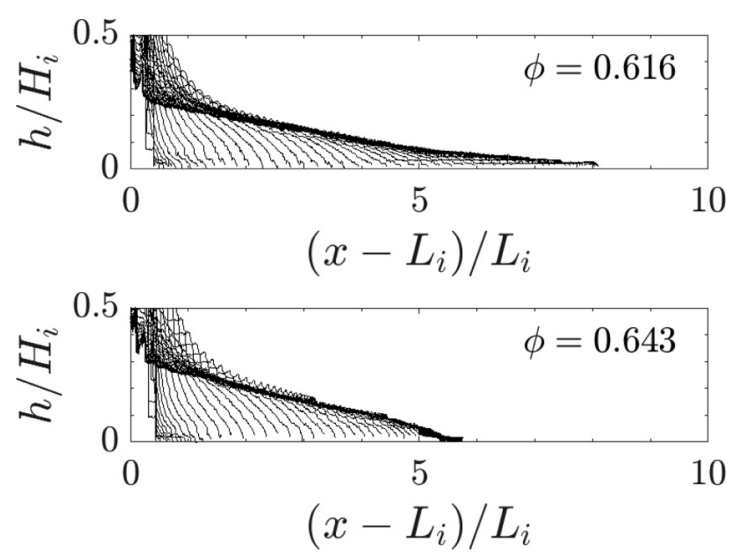

FIG. 13. Temporal evolution of the height profile $h / H_{i}$ as a function of $\left(x-L_{i}\right) / L_{i}$ for a water-saturated granular collapse ( $a=2$ and $\mathrm{St}=42$ ) with two different volume fractions, i.e., $\phi=0.616$ (top) and $\phi=0.643$ (bottom). The time step between each profile is $0.02 \mathrm{~s}$.

Figure 13 shows the temporal evolution of the height profile $h / H_{i}$ as a function of $\left(x-L_{i}\right) / L_{i}$ for $a=2$ and $\mathrm{St}=42$ with two different volume fractions, $\phi=0.616$ and $\phi=0.643$. Both deposits are of triangular shape [24]. Moreover, for $\phi=0.616$, the final deposit is more elongated with a runout length $L_{f}$ ( $\equiv L_{r 1} \equiv L_{r 2}$, here) which is larger than in the case with $\phi=0.643$.

We provide here the same quantification of mobility of the granular medium as in the previous section, i.e., $\left(L_{f}-L_{f}^{d}\right) / L_{f}^{d}$. Note that, at this value of St, no difference is obtained between the "first collapse" and "second collapse", and then $L_{f}$ corresponds to the only final spreading length. Moreover, the dry case is performed with the same experimental setup and the same grains, for $\phi \sim 0.64$ as no such significant influence of $\phi$ has been reported so far in the dry case [44]. Note that experiments performed with the present apparatus, varying $\phi$ with smaller grains (as the initial volume fraction $\phi$ can hardly be modified in a dry configuration with $d=3.15 \mathrm{~mm}$ ), suggests that the dry spreading length indeed remains roughly constant (not shown here). $\left(L_{f}-L_{f}^{d}\right) / L_{f}^{d}$ is plotted as a function $\phi$ for two initial aspect ratios, namely, $a=1$ and 2, and $\mathrm{St}=42$, in Fig. 14. $\left(L_{f}-L_{f}^{d}\right)$ is observed to increase with decreasing $\phi$, with in particular, $\left(L_{f}-L_{f}^{d}\right)$ changing sign for a critical

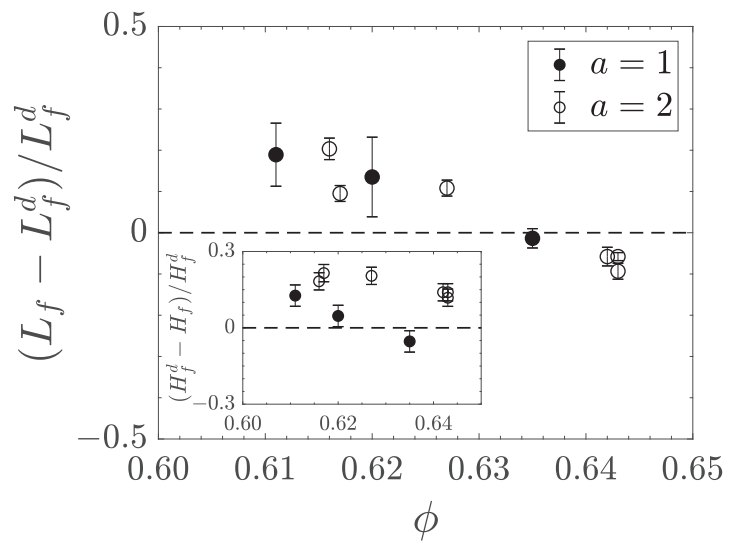

FIG. 14. Runout length $L_{f}$ relative to that of the corresponding dry collapse $L_{f}^{d}$ as a function of $\phi(\mathrm{St}=42)$ : (•) $a=1$, (O) $a=2$. Inset: corresponding final height $H_{f}$ relative to that of the dry collapse $H_{f}^{d}$. 
volume fraction $\phi_{c} \approx 0.635$. The inset presents the final height $H_{f}$ as a function of $\phi$ relative to the dry case. Despite a larger dispersion, the same trend is obtained for $a=1$ with a change of sign around $\phi_{c} \approx 0.627$. However, at $a=2, H_{f}>H_{f}^{d}$ for the whole range of $\phi$ investigated here. According to the previous observations and discussions, the difference of final shape obtained for the water-saturated configuration is attributed to the presence of the interstitial fluid. An influence of $\phi$ on the spreading length has also been reported in a fully immersed configuration [24]. However, in this case, the difference between the fully immersed case and the dry case is typically smaller than the difference observed here between the liquid-saturated case and the dry case. Moreover, this was only observed at smaller $\phi$ and at small St, i.e., in the viscous regime. Indeed, in the fully immersed configuration, the influence of $\phi$ on the collapse was mostly initiated during the first phase of the collapse, i.e., the triggering phase $I$. Here, for $\mathrm{St}=42$, the first phase is roughly nonexistent and the process inducing the difference of final length scales is therefore different, even if is also linked to the interstitial fluid. In particular, it occurs during the 'first collapse', i.e., phase $I I$. We can then conclude that the difference of the final length scales, obtained here for $\mathrm{St}=42$, can only be attributed to the specific configuration of the water-saturated granular spreading.

\section{CONCLUSION AND DISCUSSION}

Laboratory experiments of a liquid-saturated granular collapse in air have been investigated, in which various parameters were varied: the properties of the interstitial fluid and of the grains, the geometry of the initial column, and the initial compaction. Both the dynamics of the collapse and the morphology of the final deposit were characterized via the temporal evolution of the granular height profile.

First, we show that the trigger of the collapse and the shape of the final deposit are mostly controlled by the "column" Bond number, defined as Bo $=\rho g H_{i} d / \sigma$, and the ratio between the grain diameter and the capillary length $d / l_{c}$. In particular, four different regimes are observed: static, fluid-leaking, block- and continuous-avalanche regimes. The transition between these regimes are analyzed through some criteria which take capillary effects at the granular interface into account, as follows:

(1) Static regime: $\mathrm{Bo}<\mathrm{Bo}^{F}$ [see Eq. (1)]

(2) Fluid-leaking regime: $\mathrm{Bo}^{F}<\mathrm{Bo}<\mathrm{Bo}^{G}$ [see Eq. (2)]

(3) Block-avalanche regime: $\mathrm{Bo}^{G}<$ Bo and $d / l_{c}<d_{c} / l_{c}$ [see Eq. (3)]

(4) Continuous-avalanche regime: $d / l_{c}>d_{c} / l_{c}$ [see Eq. (3)] or $d / l_{c}>d_{c}^{\prime} / l_{c}$ [see Eq. 4]

The fundamental mechanism controlling these regimes is here attributed to the surface tension. However, the possible quantitative evolution of the transitions could also depend on $\phi$ and St, which cannot be addressed here with the set of experiments available. Further work is therefore needed to investigate their possible influence on each transition.

Second, we extend Lajeunesse et al. [7]'s analysis, which predicts the runout length for a dry granular collapse, to the present case of a liquid-saturated granular collapse. In particular, the present model gives an estimate of the runout length of the granular deposit as a function of the Bond number and the initial aspect ratio. The model is able to capture the main trend of the observed experimental results and the asymptotic behavior at large Bond numbers and aspect ratios. However, quantitative agreement is more difficult since the present model is derived from an order of magnitude analysis, which was already the case for dry granular collapses [7].

Finally, an investigation of the specific effect of the initial aspect ratio, the Stokes number and the initial volume fraction is performed in the continuous-avalanche regime, in which capillary effects can be disregarded. In general, the observed dynamics and final deposit of a liquid-saturated granular collapse is similar to that of a fully immersed granular collapse. A specific behavior is the split of the dynamics into two distinct front motions, referred to as "first collapse" and "second collapse", for $\mathrm{St}>\mathrm{St}_{c 1}$ with $\mathrm{St}_{c 1} \approx 0.2$, while a single viscous-dominated granular collapse is obtained, otherwise. The duration of the "first collapse" rapidly decreases for increasing St, up to $\mathrm{St}>\mathrm{St}_{c 2}$ with $\mathrm{St}_{c 2} \approx 1.5$, for which it roughly scales with the free-fall timescale $T_{\mathrm{FF}}=\left(2 H_{i} / g\right)^{1 / 2}$, 


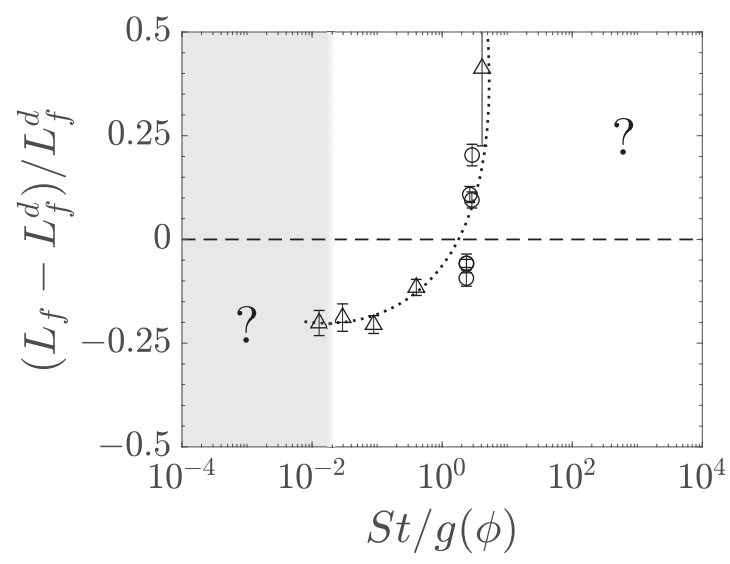

FIG. 15. Hypothetical sketch of the evolution of the final runout length $L_{f} \equiv L_{r 2}$ relative to that of the corresponding dry collapse $L_{f}^{d}$ as a function of St $/ g(\phi)$, with $g(\phi)=(1-\phi)^{-2.8}$, for liquid-saturated granular collapses in air $\left(\rho_{p} / \rho_{f} \approx 2.5\right.$ and $\left.\rho_{f} / \rho_{a}=10^{3}\right)$. Triangles and circles correspond to the runout length $L_{f} \equiv L_{r 2}$ of experiments shown in Figs. 11 and 14 with $a=2$, respectively. The gray area represents the range St $/ g(\phi) \lesssim$ $2 \times 10^{-2}$ for which no second spreading phase was observed.

usually used for dry granular collapses and dam-break flows [7,28,43]. More specifically, the dynamics of the "first collapse" is considered as a viscous-dominated regime, for $\mathrm{St}<\mathrm{St}_{c 2}$, and an inertia-dominated regime, for $\mathrm{St}>\mathrm{St}_{c 2}$. The "second collapse" corresponds to a phase in which the fluid flow drains out of the granular medium and its duration is therefore controlled by viscous dissipation. It also plays a role on the runout length of the deposit, at low St, while it can be disregarded, at large St. In any case, the characteristics of the final deposit, i.e., the runout length and the final height, follow simple power-laws over $a$ with a prefactor depending on St and $\phi$.

More surprisingly, we show that the interstitial fluid can either play a dissipative or a driving role on the collapse depending on both the Stokes number St and the initial volume fraction $\phi$. In particular, a driving role of the interstitial fluid on the runout of the granular media is observed for $\phi<0.635$ and $\mathrm{St}=42$. Note that to discuss in details the influence of St and $\phi$ in the previous sections, we kept other dimensionless parameters constant in each case to avoid misinterpretation of the results. Some set of experiments performed here have thus been disregarded so far.

We propose in the following a discussion on the driving role of the interstitial fluid that could be of interest for geophysical applications and that would unify the entire set of experiments in the continuous-avalanche regime. Results obtained in the previous sections suggest that viscous dissipation decreases for St increasing and/or $\phi$ decreasing. While the viscous dissipation is overcome, the interstitial fluid could play a driving role, which has only be observed so far varying $\phi$ at $\mathrm{St}=42$. To consider both $\mathrm{St}$ and $\phi$ on this influence, Fig. 15 shows the dimensionless final runout length $\left(L_{f}-L_{f}^{d}\right) / L_{f}^{d} \equiv\left(L_{r 2}-L_{f}^{d}\right) / L_{f}^{d}$ as a function of $\mathrm{St} / g(\phi)$, for the set of experiments corresponding to the results shown in Fig. 11 (triangles) and Fig. 14 (circles) and another set of experiments which have not been reported yet. The function $g(\phi)=(1-\phi)^{-2.8}$, already mentioned and used in Sec. VI B, allows to account for $\phi$ into an effective viscosity of an equivalent surrounding fluid seen by an individual particle into the liquid-saturated medium [42]. Then, St $/ g(\phi)$ is nothing but a Stokes number based on this effective viscosity instead of the fluid viscosity $\eta_{f}$. In this scenario, $\phi$ only affects the dynamics through a modification of the viscous dissipation term.

The runout length $\left(L_{f}-L_{f}^{d}\right) / L_{f}^{d}$ is shown to increase with increasing St $/ g(\phi)$. More specifically, $\left(L_{f}-L_{f}^{d}\right)$ changes sign, for $\mathrm{St} / g(\phi) \approx 1$, which indicates a transition from a dissipative role to a driving role of the interstitial fluid on the collapse of the granular material. A possible explanation is that, at low St $/ g(\phi)$, namely, St $/ g(\phi)<1$, the granular material is slowed down by the interstitial fluid due to viscous dissipation leading to $\left(L_{f}-L_{f}^{d}\right)<0$. Conversely, at larger St $/ g(\phi)$, i.e., 
$\mathrm{St} / g(\phi)>1$, the fluid may even make easier the granular flow through a drag force in the direction of the flow or a decrease of frictional contacts.

Finally, the proposed trend is represented in Fig. 15 with a dotted line. Other dedicated experiments, beyond the scope of the present paper, would be useful to verify the pertinence of the proposed evolution of the liquid-saturated granular collapse induced by a driving role of the interstitial fluid. In particular, the existence of a local maximum of the spreading length at a critical value of St $/ g(\phi)$ has to be investigated. Note that the grain-fluid density ratio $\rho_{p} / \rho_{f}$ is also an important parameter in this problem, which has not been varied in the present work (recall that $\rho_{p} / \rho_{f} \approx 2.5$ and $\rho_{f} / \rho_{a}=10^{3}$ ). Further work is therefore necessary to assess the influence of this parameter on the dynamics of the collapse and the shape of the final deposit.

\section{ACKNOWLEDGMENTS}

This work was funded by the Agence Française pour la Biodiversité. We thank P. Aussillous and G. Ovarlez for their constructive remarks on the analytical model. A. Bougouin is grateful to N. Malik and M. Saint-Martin for preliminary experiments performed during their internship.

\section{APPENDIX A: MEASUREMENTS OF THE AVALANCHE AND REPOSE ANGLES}

The angle of avalanche $\theta_{a}$ and the angle of repose $\theta_{r}$ of grains (except for $d=5.0$ and $10.0 \mathrm{~mm}$ ) were estimated from the variations of the slope of an initially horizontal plane granular bed in a box $\left(20 \times 10 \times 5 \mathrm{~cm}^{3}\right)$ which is slowly tilted. Note that the box width to particle diameter ratio was always $>30$ which ensured that there was no lateral wall effect [45]. Figure 16 presents the angles $\theta_{a}$ and $\theta_{r}$ as a function of the grain diameter $d$. A similar trend is observed for both angles. In particular, $\theta$ decreases while $d$ is increased and then, for $d \gtrsim D=550 \mu \mathrm{m}$, it reaches a constant value $\langle\theta\rangle$ (dashed lines). In the following, the $d$-dependency is approximated by a relation of the type $\theta /\langle\theta\rangle=f(d / D)$ (inset of Fig. 16). In the present work, we propose

$$
\frac{\theta}{\langle\theta\rangle}=\mid \begin{array}{ll}
1-0.2 \ln (d / D) & \begin{array}{l}
\text { for } \mathrm{d} / \mathrm{D}<1, \\
\text { otherwise, }
\end{array}
\end{array}
$$

where the averaged value of the avalanche and repose angles are set to $\left\langle\theta_{a}\right\rangle=28^{\circ}$ and $\left\langle\theta_{r}\right\rangle=22^{\circ}$, at large $d$. Note that the choice of this relation is arbitrary and other relations could be used.

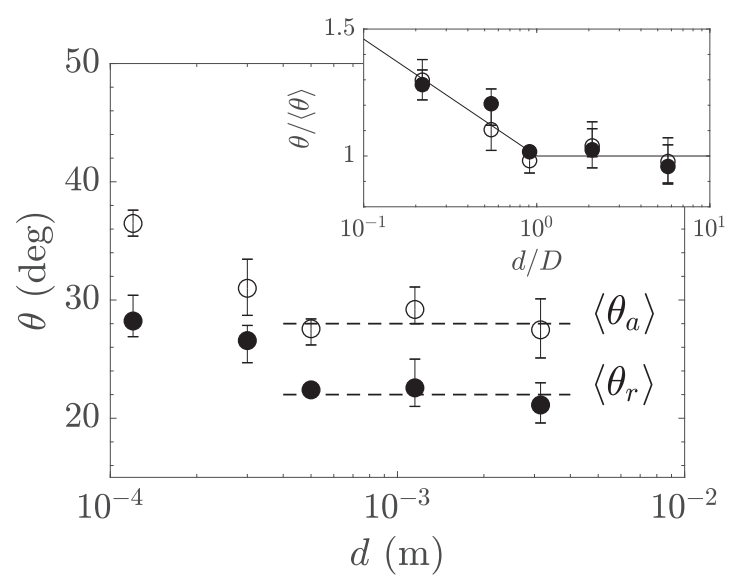

FIG. 16. Angle of avalanche $\theta_{a}(\mathrm{O})$ and angle of repose $\theta_{r}(\bullet)$ as a function of the grain diameter $d$; (- - -) Mean values $\left\langle\theta_{a}\right\rangle=28^{\circ}$ and $\left\langle\theta_{r}\right\rangle=22^{\circ}$, for $d>D=550 \mu \mathrm{m}$, used in Eq. (A1). Inset: Avalanche and repose angles scaled by the mean angle as a function of $d / D$; (-) Eq. (A1). 


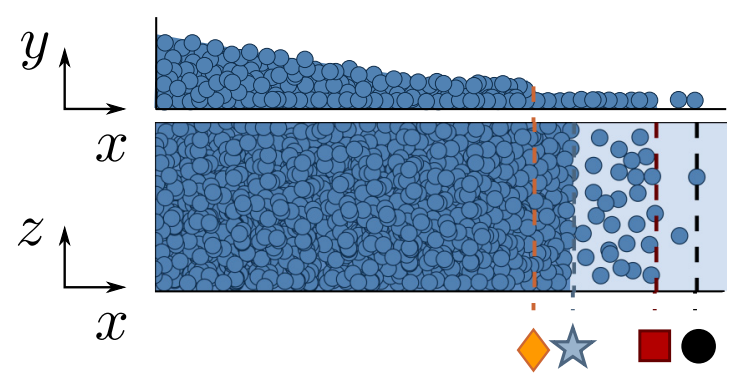

FIG. 17. Sketch of the final deposit of a liquid-saturated granular collapse with different estimates of the runout $L_{f}$ ( $\equiv L_{r 2}$ defined in Fig. 7): $\diamond$ height front of two grain diameters, $\star$ quasi-2D front and all grains in contact, $\square$ quasi-2D front, and $\bullet$ farthest grain.

\section{APPENDIX B: DETERMINATION OF THE RUNOUT LENGTH IN THE EXPERIMENTS}

In this work, a specific attention has been drawn to the estimation of the runout length of the final deposit. In particular, four criteria have been defined (Fig. 17): a height front of two grain diameters $(\diamond)$, a quasi-2D front and all grains in contact $(\star)$, a quasi-2D front $(\square)$, and the farthest grain $(\bullet)$. In the paper, the runout length $L_{f}$ ( $\equiv L_{r 2}$ defined in Fig. 7) corresponds to the mean of values obtained with these different criteria. Note that, for $d \leqslant 1.15 \mathrm{~mm}$, the four criteria of the runout length are strictly equivalent and this specific procedure can be omitted. Same conclusions can also be done for the determination of the rest length $L_{r 1}$ (Fig. 7 for the definition).

Figure 18 shows the dimensionless runout difference $\left(L_{f}-L_{f}^{d}\right) / L_{f}^{d}$, where $L_{f}^{d}$ is the runout length of the equivalent dry collapse, as a function of the Stokes number St [Fig. 18(a)] and the initial volume fraction $\phi$ [Fig. 18(b)] using different criteria defined in Fig. 17. It can be observed that trends are respected for each criterion. In particular, $\left(L_{f}-L_{f}^{d}\right) / L_{f}^{d}$ increases for St increasing and for $\phi$ decreasing, according to previous conclusions. However, some quantitative differences are obtained between different criteria showing the difficulties to define the runout length, precisely, in the liquid-saturated granular configuration. In particular, it can be noted that the inaccuracy is more important at low $\phi$.
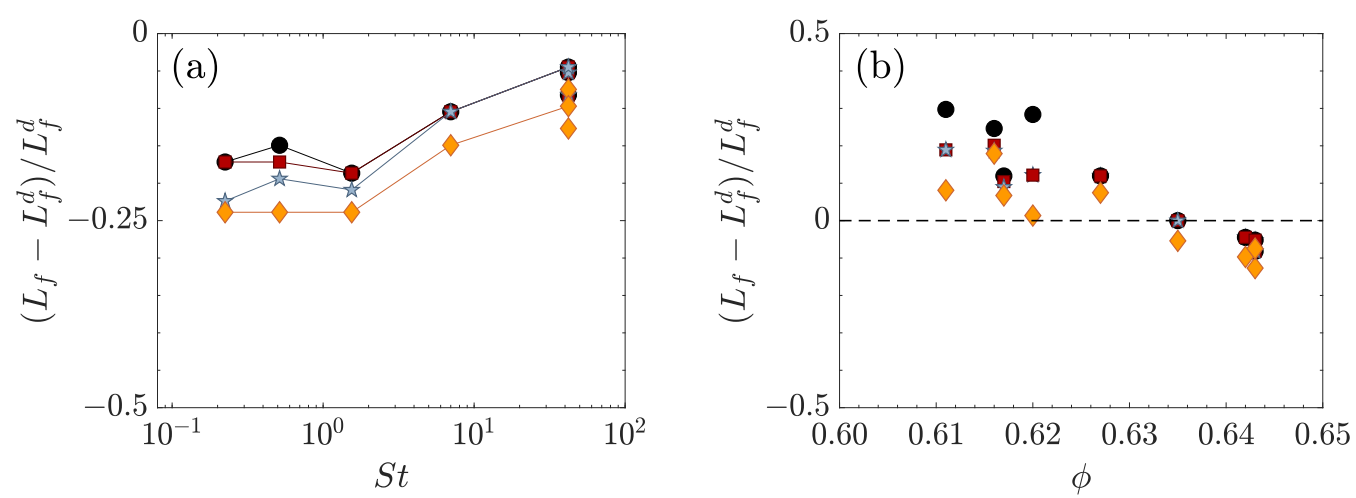

FIG. 18. Runout length $L_{f}$ ( $\equiv L_{r 2}$ defined in Fig. 7) relative to that of the corresponding dry collapse $L_{f}^{d}$ as a function of (a) the Stokes number St and (b) the initial volume fraction $\phi$ using different criteria defined in Fig. 17. 


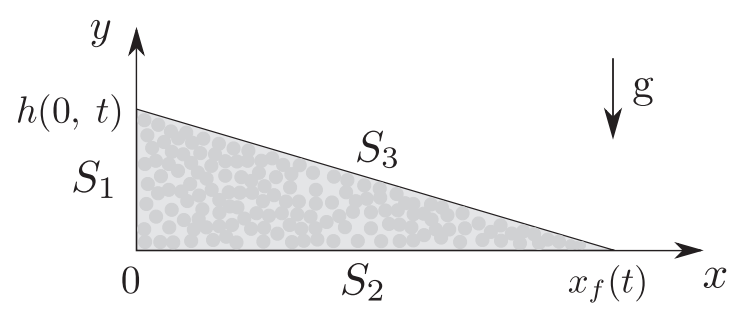

FIG. 19. Sketch of the collapse of a liquid-saturated granular mass on a horizontal plane at an arbitrary time.

\section{APPENDIX C: EXTENDING LAJEUNESSE ET AL. [7]'S MODEL}

Let us first consider a liquid-saturated granular mass spreading on a horizontal plane (Fig. 19). In line with experimental observations, the shape of the height profile is assumed to be triangular which is in fairly good agreement in the continuous-avalanche regime and, at least at first order, in the block-avalanche regime. During the collapse, momentum conservation reduces to a force balance on the fluid-granular mixture in the horizontal direction $x$, in particular, with a force $F_{S_{1}}$ induced by gravity on the vertical surface $S_{1}$ and a friction force $F_{S_{2}}$ applied to the bottom surface $S_{2}$. For simplicity, the air pressure is neglected here [note that we verified that the solution of Eq. (C5) remains unchanged if one takes the air pressure into account]. One therefore obtains

$$
\rho H_{i} L_{i} \frac{d^{2} x_{f}(t)}{d t^{2}}=F_{S_{1}}-F_{S_{2}}
$$

where $x_{f}(t)$ represents the instantaneous front position. Note that the triangular shape leads to the expression $h(x, t)=h(0, t)\left[1-x / x_{f}(t)\right]$. Assuming that the force $F_{S_{1}}$ corresponds to the hydrostatic pressure of the grain-fluid mixture on surface $S_{1}$, one gets

$$
F_{S_{1}}=\int_{0}^{h(0, t)} \rho g[h(0, t)-y] d y=\frac{\rho g h(0, t)^{2}}{2} .
$$

The friction force $F_{S_{2}}$ is assumed to be induced by the normal granular stress on the bottom surface $S_{2}$ through the Coulomb law. This normal stress includes both the local granulostatic pressure $P_{g}=\phi\left(\rho_{p}-\rho_{f}\right) g h$ and the capillary pressure $P_{c} \approx \varepsilon \sigma / d$. This reads

$$
F_{S_{2}}=\int_{0}^{x_{f}(t)} \mu\left[\phi\left(\rho_{p}-\rho_{f}\right) g h(x, t)+\varepsilon \frac{\sigma}{d}\right] d x=\mu\left[\frac{\phi\left(\rho_{p}-\rho_{f}\right) g h(0, t)}{2}+\varepsilon \frac{\sigma}{d}\right] x_{f}(t),
$$

where $\mu$ is the friction coefficient between the granular material and the bottom plane. It can be noted that we assumed that the capillary pressure is fully transmitted to the granular material. Using Eqs. (C2) and (C3) and the mass conservation to eliminate $h(0, t)$ in Eq. (C1), i.e., $2 H_{i} L_{i}=h(0, t) x_{f}(t)$, one obtains the following differential equation for $x_{f}(t)$ :

$$
\frac{d^{2} x_{f}(t)}{d t^{2}}=\frac{2 g H_{i} L_{i}}{x_{f}^{2}(t)}-\frac{\mu \phi\left(\rho_{p}-\rho_{f}\right) g}{\rho}-\frac{\mu \varepsilon \sigma}{\rho H_{i} L_{i} d} x_{f}(t) .
$$

This equation can be viewed as an extension for a liquid-saturated granular collapse of Lajeunesse et al. [7]'s analysis done for a dry collapse, because of the presence of the last term in Eq. (C4) which is proportional to surface tension. Following Lajeunesse et al. [7] instead of solving Eq. (C4), we rather perform a scaling analysis of the different terms.

We take as timescale the following free-fall time $\tau_{c} \equiv \lambda T_{\mathrm{FF}}$, where $T_{\mathrm{FF}}=\left(2 H_{i} / g\right)^{1 / 2}$ is the freefall timescale defined at the column scale and usually used in dry granular collapses and dam-break flows $[7,28,43]$. The parameter $\lambda$ is an empirical constant, which can be viewed as a dimensionless collapse time. This constant is estimated from the present experiments of a water-saturated granular collapse (Fig. 20). More specifically, we first estimate the time $T_{r 1}$ as the time when the granular 


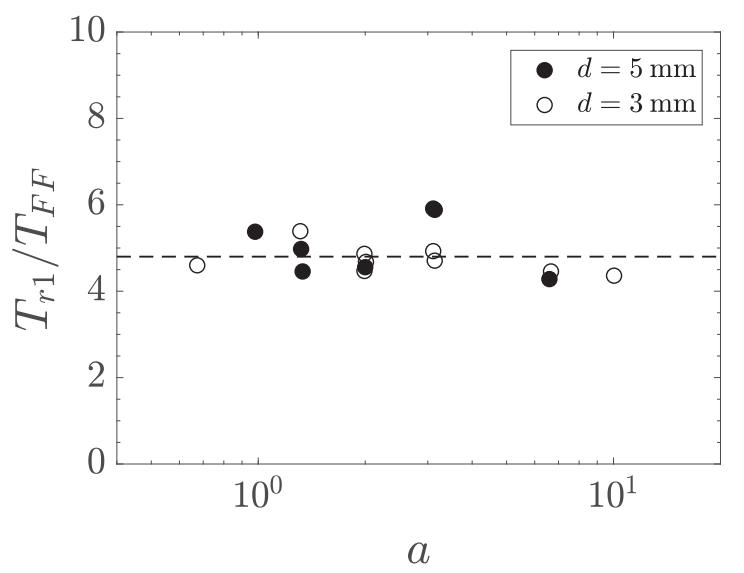

FIG. 20. Collapse time $\lambda=T_{r 1} / T_{\mathrm{FF}}$ (defined in Fig. 7) as a function of the initial aspect ratio $a$ for (O) $d=3.15 \mathrm{~mm}$ and $(\bullet) d=5.0 \mathrm{~mm}$. Here, time is normalized by the free-fall timescale $T_{\mathrm{FF}}=\left(2 H_{i} / g\right)^{1 / 2}$. The dashed lines corresponds to the mean value of $\lambda=4.8$.

front reaches the runout length $L_{r 1}$ defined in Fig. 7. Note that, for large St, we have $L_{r 1} \equiv L_{r 2} \equiv L_{f}$ and the time $T_{r 1}$ corresponds to the timescale of the granular collapse. Figure 20 shows the time $T_{r 1}$ of a water-saturated granular collapse normalized by the free-fall timescale $T_{\mathrm{FF}}=\left(2 H_{i} / g\right)^{1 / 2}$ as a function of the initial aspect ratio $a=H_{i} / L_{i}$, for $d=3.15 \mathrm{~mm}$ (opened circles) and $d=5.0 \mathrm{~mm}$ (closed circles). Recall that the time $T_{\mathrm{FF}}$ is the time that a single grain need to travel over a distance length $H_{i}$ in free-fall. Figure 20 indicates that $T_{r 1} \propto T_{\mathrm{FF}}$, in particular, we find $T_{r 1} / T_{\mathrm{FF}} \approx 4.8$ and we will therefore set this value.

Let us thus assume that the collapse occurs during a time $t \approx \tau_{c}$ and use as length scale for the front location and the front displacement $L_{f}$ and $L_{f}-L_{i}$, respectively. One can write $x_{f}\left(\tau_{c}\right) \approx$ $L_{f}$ and $d^{2} x_{f}\left(\tau_{c}\right) / d t^{2} \approx\left(L_{f}-L_{i}\right) / \tau_{c}^{2}$. Using these approximations and the definition of the initial aspect ratio $a=H_{i} / L_{i}$ and that of the Bond number Bo $=\rho g H_{i} d / \sigma$, Eq. (C4) can be rewritten so as to give the proposed model,

$$
\frac{L_{f}-L_{i}}{L_{i}}=4 \lambda^{2} a^{2}\left(\frac{L_{f}}{L_{i}}\right)^{-2}-2 \lambda^{2} \mu \phi \frac{\rho_{p}-\rho_{f}}{\rho} a-2 \varepsilon \lambda^{2} \mu \frac{a}{\mathrm{Bo}}\left(\frac{L_{f}}{L_{i}}\right) .
$$

[1] R. M. Iverson, The physics of debris flows, Rev. Geophys. 35, 245 (1997).

[2] M. Jakob and O. Hungr, Debris-flow Hazards and Related Phenomena (Springer, Berlin, 2005), p. 739.

[3] D. Rickenmann, Empirical relationships for debris flows, Nat. Hazards 19, 47 (1999).

[4] G. Lube, H. E. Huppert, R. S. J. Sparks, and M. A. Hallworth, Axisymmetric collapses of granular columns, J. Fluid Mech. 508, 175 (2004).

[5] E. Lajeunesse, A. Mangeney-Castelnau, and J. P. Vilotte, Spreading of a granular mass on a horizontal plane, Phys. Fluids 16, 2371 (2004).

[6] G. Lube, H. E. Huppert, R. S. J. Sparks, and A. Freundt, Collapses of two-dimensional granular columns, Phys. Rev. E 72, 041301 (2005).

[7] E. Lajeunesse, J. B. Monnier, and G. M. Homsy, Granular slumping on a horizontal surface, Phys. Fluids 17, 103302 (2005).

[8] N. J. Balmforth and R. R. Kerswell, Granular collapse in two dimensions, J. Fluid Mech. 538, 399 (2005).

[9] L. Staron and E. J. Hinch, The spreading of a granular mass: Role of grain properties and initial conditions, Granular Matter 9, 205 (2007).

[10] N. Mitarai and F. Nori, Wet granular materials, Adv. Phys. 55, 1 (2006). 
[11] D. J. Hornbaker, R. Albert, I. Albert, A.-L. Barabási, and P. Schiffer, What keeps sandcastles standing? Nature 387, 765 (1997).

[12] M. Scheel, R. Seemann, M. Brinkmann, M. Di Michiel, A. Sheppard, B. Breidenbach, and S. Herminghaus, Morphological clues to wet granular pile stability, Nature 7, 189 (2008).

[13] M. Pakpour, M. Habibi, P. Møller, and D. Bonn, How to construct the perfect sandcastle, Sci. Rep. 2, 549 (2012).

[14] R. Artoni, A. C. Santomaso, F. Gabrieli, D. Tono, and S. Cola, Collapse of quasi-two-dimensional wet granular columns, Phys. Rev. E 87, 032205 (2013).

[15] F. Gabrieli, R. Artoni, A. Santomaso, and S. Cola, Discrete particle simulations and experiments on the collapse of wet granular columns, Phys. Fluids 25, 103303 (2013).

[16] V. J. Langlois, A. Quiquerez, and P. Allemand, Collapse of a two-dimensional brittle granular column: Implications for understanding dynamic rock fragmentation in a landslide, J. Geophys. Res. Earth Surf. 120, 1866 (2015).

[17] E. Fern and K. Soga, Granular column collapse of wet sand, Procedia Eng. 175, 14 (2017).

[18] A. C. Santomaso, S. Volpato, and F. Gabrieli, Collapse and runout of granular columns in pendular state, Phys. Fluids 30, 063301 (2018).

[19] C. Bonnoit, D. Darnige, E. Clement, and A. Lindner, Inclined plane rheometry of a dense granular suspension, J. Rheol. 54, 65 (2010).

[20] C. Ancey, N. Andreini, and G. Epely-Chauvin, The dam-break problem for concentrated suspensions of neutrally buoyant particles, J. Fluid Mech. 724, 95 (2013).

[21] C. Ancey, N. Andreini, and G. Epely-Chauvin, Granular suspension avalanches. I. Macro-viscous behavior, Phys. Fluids 25, 033301 (2013).

[22] N. Andreini, C. Ancey, and G. Epely-Chauvin, Granular suspension avalanches. II. Plastic regime, Phys. Fluids 25, 033302 (2013).

[23] A. Bougouin, L. Lacaze, and T. Bonometti, Collapse of a neutrally buoyant suspension column: From Newtonian to apparent non-Newtonian flow regimes, J. Fluid Mech. 826, 918 (2017).

[24] L. Rondon, O. Pouliquen, and P. Aussillous, Granular collapse in a fluid: Role of the initial volume fraction, Phys. Fluids 23, 073301 (2011).

[25] V. Topin, Y. Monerie, F. Perales, and F. Radjaï, Collapse Dynamics and Runout of Dense Granular Materials in a Fluid, Phys. Rev. Lett. 109, 188001 (2012).

[26] C. Wang, Y. Wang, C. Peng, and X. Meng, Dilatancy and compaction effects on the submerged granular column collapse, Phys. Fluids 29, 103307 (2017).

[27] K. Kumar, J.-Y. Delenne, and K. Soga, Mechanics of granular column collapse in fluid at varying slope angles, J. Hydrodyn. 29, 529 (2017).

[28] A. Bougouin and L. Lacaze, Granular collapse in a fluid: Different flow regimes for an initially densepacking, Phys. Rev. Fluids 3, 064305 (2018).

[29] Z. Zheng, H. E. Huppert, N. M. Vriend, J. A. Neufeld, and P. F. Linden, Flow of buoyant granular materials along a free surface, J. Fluid Mech. 848, 312 (2018).

[30] L. Jing, G. C. Yang, C. Y. Kwok, and Y. D. Sobral, Dynamics and scaling laws of underwater granular collapse with varying aspect ratios, Phys. Rev. E 98, 042901 (2018).

[31] S. Courrech du Pont, P. Gondret, B. Perrin, and M. Rabaud, Granular Avalanches in Fluids, Phys. Rev. Lett. 90, 044301 (2003).

[32] L. Jing, G. C. Yang, C. Y. Kwok, and Y. D. Sobral, Flow regimes and dynamic similarity of immersed granular collapse: A CFD-DEM investigation, Powder Technol. 345, 532 (2019).

[33] M. K. C. Sridhar and C. R. Reddy, Surface tension of polluted waters and treated wastewater, Environ. Pollut. 7, 49 (1984).

[34] P. Philippe and D. Bideau, Compaction dynamics of a granular medium under vertical tapping, Europhys. Lett. 60, 677 (2002).

[35] I. R. Ionescu, A. Mangeney, F. Bouchut, and O. Roche, Viscoplastic modeling of granular column collapse with pressure-dependent rheology, J. Non-Newtonian Fluid Mech. 219, 1 (2015).

[36] See Supplemental Material at http://link.aps.org/supplemental/10.1103/PhysRevFluids.4.124306, corresponding to water-saturated granular collapses in air, for $d=120 \mu \mathrm{m}$ (static regime), 
$d=500 \mu \mathrm{m} \quad$ (block-avalanche regime), and $d=5 \mathrm{~mm} \quad$ (continuous-avalanche regime) with $a \approx 1.3\left(H_{i}=20 \mathrm{~cm}, L_{i}=15 \mathrm{~cm}\right)$, and $d=300 \mu \mathrm{m}$ (fluid-leaking regime) with $a \approx 2\left(H_{i}=\right.$ $\left.29.7 \mathrm{~cm}, L_{i}=15 \mathrm{~cm}\right)$.

[37] J.-Y. Delenne, V. Richefeu, and F. Radjai, Liquid clustering and capillary pressure in granular media, J. Fluid Mech. Rapids 762, R5 (2015).

[38] R. Fisher, On the capillary forces in a ideal soil: Correction of formulae given by W. B. Haines, J. Agric. Sci. 16, 492 (1926).

[39] F. M. Orr, L. E. Scriven, and A. P. Rivas, Pendular rings between solids: Meniscus properties and capillary force, J. Fluid Mech. 67, 723 (1975).

[40] T. C. Halsey and A. J. Levine, How Sandcastles Fall, Phys. Rev. Lett. 80, 3141 (1998).

[41] V. Topin, F. Dubois, F. Perales, and A. Wachs, Micro-rheology of dense particulate flows: Application to immersed avalanches, J. Non-Newtonian Fluid Mech. 166, 63 (2011).

[42] L. G. Gibilaro, K. Gallucci, R. Di Felice, and P. Pagliai, On the apparent viscosity of a fluidized bed, Chem. Eng. Sci. 62, 294 (2006).

[43] O. Roche, S. Montserrat, Y. Nino, and A. Tamburrino, Experimental observations of water-like behavior of initially fluidized, dam break granular flows and their relevance for the propagation of ash-rich pyroclastic flows, J. Geophys. Res. Solid Earth 113, B12203 (2008).

[44] A. Daerr and S. Douady, Sensitivity of granular surface flows to preparation, Europhys. Lett. 47, 324 (1999).

[45] S. Courrech du Pont, P. Gondret, B. Perrin, and M. Rabaud, Wall effects on granular heap stability, Europhys. Lett. 61, 492 (2003). 